\title{
THE ALPERIN AND UNO CONJECTURES FOR THE FISCHER SIMPLE GROUP $\mathrm{Fi}_{22}$
}

\author{
Jianbei An E.A. O'Brien
}

\begin{abstract}
Using the local subgroup strategy of [3] and [4], we classify the radical subgroups and chains of the Fischer simple group $\mathrm{Fi}_{22}$ and verify the Alperin weight conjecture and the Uno reductive conjecture for this group; the latter is a refinement of the Dade reductive and Isaacs-Navarro conjectures.
\end{abstract}

\section{Introduction}

Let $G$ be a finite group, $p$ a prime and $B$ a $p$-block of $G$. Alperin [1] conjectured that the number of $B$-weights equals the number of irreducible Brauer characters of $B$. Dade [11] generalized the Knörr-Robinson version of the Alperin weight conjecture and presented his ordinary conjecture exhibiting the number of ordinary irreducible characters of a fixed defect in $B$ in terms of an alternating sum of related values for $p$-blocks of some $p$-local subgroups of $G$. Dade [12] presented several other forms of his conjecture and announced that his final conjecture needs only to be verified for finite non-abelian simple groups; in addition, if a finite group has a cyclic outer automorphism group, then the projective invariant conjecture is equivalent to the final conjecture. Recently, Isaacs and Navarro [16] proposed a new conjecture which is a refinement of the Alperin-McKay conjecture, and Uno [19] raised an alternating sum version of the Isaacs-Navarro conjecture which is a refinement of the Dade conjecture.

In [3] and [4], we presented a local subgroup strategy to decide the Alperin and Dade conjectures for the finite simple groups and demonstrated its computational effectiveness by using it to verify the Alperin and Dade conjectures for the Conway simple group $\mathrm{Co}_{2}$ and the Fischer simple group $\mathrm{Fi}_{23}$. In this paper, we apply the strategy to verify the Alperin and Uno conjectures for the Fischer simple group $\mathrm{Fi}_{22}$. Although the outlines of our computations and proofs are similar to those of the Alperin and Dade conjectures for $\mathrm{Co}_{2}$ and $\mathrm{Fi}_{23}$, the details are significantly more complex, since we must verify Uno's invariant conjecture for $\mathrm{Fi}_{22}$, the projective invariant conjecture

This work was supported in part by the Marsden Fund of New Zealand via grant UOA 124. 1991 Mathematics Subject Classification. Primary 20C20, 20C34, 20C08. Keywords, representations of finite groups, Fischer's simple groups, Dade's conjecture. 
for the 2-covering group $2 . \mathrm{Fi}_{22}$, and the projective conjecture for the 3 - and 6 -covering groups, $3 . \mathrm{Fi}_{22}$ and $6 . \mathrm{Fi}_{22}$.

The challenge in deciding the conjectures for these groups is to determine the character tables of the normalizers of the radical 2- and 3-chains. Our approach to determining the character tables of the normalizers is similar to that employed in $\mathrm{Fi}_{23}$, but the calculations are more complicated because of the large degrees, 185328 and 370656 , of the faithful permutation representations of $3 . \mathrm{Fi}_{22}$ and $6 . \mathrm{Fi}_{22}$. If a relevant normalizer is a maximal subgroup of a covering group, then its character table is usually available in a character table library supplied with the computational algebra system GAP [18]. Otherwise, we constructed a "useful" representation of the normalizer and attempted to compute directly its character table; if this construction failed, we used lifting of characters of quotient groups, and induction and decomposition of characters of subgroups of the normalizer to obtain its character table. We present the details in Section 6.

The paper is organized as follows. In Section 2, we fix notation and state the Alperin weight conjecture, the Isaacs-Navarro conjecture, the Dade and Uno invariant, projective, and projective invariant conjectures, and state a useful lemma. In Section 3 , we recall our (modified) local subgroup strategy and explain how we applied it to determine the radical subgroups of $\mathrm{Fi}_{22}$. In Section 4, we classify the radical subgroups of $\mathrm{Fi}_{22}$ up to conjugacy and verify the Alperin weight conjecture. The classification of radical 2-subgroups of $\mathrm{Fi}_{22}$ was given in [4, p. 631]; we report these in detail in Section 4 because many subgroups there will be used in the next section to construct radical 2-chains. In Section 5, we do some cancellations in the alternating sum of Uno's conjecture when $p=2$ or 3 , and then determine radical chains (up to conjugacy) and their local structures. In Section 6, we verify Uno's invariant conjecture for $\mathrm{Fi}_{22}$. In Section 7 , we verify Uno's projective invariant conjecture for $2 . \mathrm{Fi}_{22}$, and in Sections 8 and 9 , we verify Uno's projective conjecture for $3 . \mathrm{Fi}_{22}$ and $6 . \mathrm{Fi}_{22}$, respectively.

\section{The conjectures and a lemma}

Let $R$ be a $p$-subgroup of a finite group $G$. Then $R$ is radical if $O_{p}(N(R))=R$, where $O_{p}(N(R))$ is the largest normal $p$-subgroup of the normalizer $N(R)=N_{G}(R)$. Denote by $\operatorname{Irr}(G)$ the set of all irreducible ordinary characters of $G$, and let $\operatorname{Blk}(G)$ be the set of $p$-blocks, $B \in \operatorname{Blk}(G)$ and $\varphi \in \operatorname{Irr}(N(R) / R)$. The pair $(R, \varphi)$ is called a $B$-weight if $\mathrm{d}(\varphi)=0$ and $B(\varphi)^{G}=B$ (in the sense of Brauer), where $\mathrm{d}(\varphi)=\log _{p}\left(|G|_{p}\right)-\log _{p}\left(\varphi(1)_{p}\right)$ is the $p$-defect of $\varphi$ and $B(\varphi)$ is the block of $N(R)$ containing $\varphi$. A weight is always identified with its $G$-conjugates. Let $\mathcal{W}(B)$ be the number of $B$-weights, and $\ell(B)$ the number of irreducible Brauer characters of $B$. Alperin conjectured that $\mathcal{W}(B)=\ell(B)$ for each $B \in \operatorname{Blk}(G)$.

Given a $p$-subgroup chain

$$
C: P_{0}<P_{1}<\cdots<P_{n}
$$

of $G$, define $|C|=n$, the $k$-th subchain $C_{k}: P_{0}<P_{1}<\cdots<P_{k}$, and

$$
N(C)=N_{G}(C)=N\left(P_{0}\right) \cap N\left(P_{1}\right) \cap \cdots \cap N\left(P_{n}\right) .
$$


The chain $C$ is radical if it satisfies the following two conditions:

(a) $P_{0}=O_{p}(G)$ and (b) $P_{k}=O_{p}\left(N\left(C_{k}\right)\right)$ for $1 \leq k \leq n$.

Denote by $\mathcal{R}=\mathcal{R}(G)$ the set of all radical $p$-chains of $G$. Let $B \in \operatorname{Blk}(G)$ and let $D(B)$ be a defect group of $B$. The $p$-local rank (see [2]) of $B$ is the number

$$
\operatorname{plr}(B)=\max \left\{|C|: C \in \mathcal{R}, C: P_{0}<P_{1}<\cdots<P_{n} \leq D(B)\right\} .
$$

Let $Z$ be a cyclic group and $\hat{G}=Z$. $G$ a central extension of $Z$ by $G$, and $C \in \mathcal{R}(G)$. Denote by $N_{\hat{G}}(C)$ the preimage $\eta^{-1}(N(C))$ of $N(C)$ in $\hat{G}$, where $\eta$ is the natural group homomorphism from $\hat{G}$ onto $G$ with kernel $Z$. Let $\rho$ be a faithful linear character of $Z$ and $\hat{B}$ a block of $\hat{G}$ covering the block $B(\rho)$ of $Z$ containing $\rho$. Denote by $\operatorname{Irr}\left(N_{\hat{G}}(C), \hat{B}, d, \rho\right)$ the set of irreducible characters $\psi$ of $N_{\hat{G}}(C)$ such that $\psi$ lies over $\rho, \mathrm{d}(\psi)=d$ and $B(\psi)^{\hat{G}}=\hat{B}$ and set $\mathrm{k}\left(N_{\hat{G}}(C), \hat{B}, d, \rho\right)=\left|\operatorname{Irr}\left(N_{\hat{G}}(C), \hat{B}, d, \rho\right)\right|$.

Dade's Projective Conjecture [12]. If $O_{p}(G)=1$ and $\hat{B}$ is a $p$-block of $\hat{G}$ covering $B(\rho)$ with defect group $D(\hat{B}) \neq O_{p}(Z)$, then

$$
\sum_{C \in \mathcal{R} / G}(-1)^{|C|} \mathrm{k}\left(N_{\hat{G}}(C), \hat{B}, d, \rho\right)=0,
$$

where $\mathcal{R} / G$ is a set of representatives for the $G$-orbits of $\mathcal{R}$.

Let $\hat{H}$ be a subgroup of a finite group $\hat{G}, \varphi \in \operatorname{Irr}(\hat{H})$ and let $r(\varphi)=r_{p}(\varphi)$ be the integer $0<r(\varphi) \leq(p-1)$ such that the $p^{\prime}$-part $(|\hat{H}| / \varphi(1))_{p^{\prime}}$ of $|\hat{H}| / \varphi(1)$ satisfies

$$
\left(\frac{|\hat{H}|}{\varphi(1)}\right)_{p^{\prime}} \equiv r(\varphi) \quad(\bmod p) .
$$

Given $1 \leq r \leq(p-1) / 2$, let $\operatorname{Irr}(\hat{H},[r])$ be the subset of $\operatorname{Irr}(\hat{H})$ consisting of characters $\varphi$ such that $r(\varphi) \equiv \pm r \quad(\bmod p)$, and $\operatorname{let} \operatorname{Irr}(\hat{H}, \hat{B}, d, \rho,[r])=\operatorname{Irr}(\hat{H}, \hat{B}, d, \rho) \cap \operatorname{Irr}(\hat{H},[r])$ and $\mathrm{k}(\hat{H}, \hat{B}, d, \rho,[r])=|\operatorname{Irr}(\hat{H}, \hat{B}, d, \rho,[r])|$.

Suppose $Z=1$ and let $\hat{B}=B \in \operatorname{Blk}(G)$ with a defect group $D=D(B)$ and the Brauer correspondent $b \in \operatorname{Blk}\left(N_{G}(D)\right)$. Then $\mathrm{k}(N(D), B, d(B),[r])$ is the number of characters $\varphi \in \operatorname{Irr}(b)$ such that $\varphi$ has height 0 and $r(\varphi) \equiv \pm r \quad(\bmod p)$, where $\mathrm{d}(B)$ is the defect of $B$.

Isaacs-Navarro Conjecture [16, Conjecture B]. In the notation above,

$$
\mathrm{k}(G, B, \mathrm{~d}(B),[r])=\mathrm{k}(N(D), B, \mathrm{~d}(B),[r]) .
$$

The following refinement of Dade's conjecture is due to Uno.

Uno's Projective Conjecture [19, Conjecture 3.2]. If $O_{p}(G)=1$ and if $D(\hat{B}) \neq$ $O_{p}(Z)$, then for all integers $d \geq 0$, faithful $\rho \in \operatorname{Irr}(Z)$ and $1 \leq r \leq(p-1) / 2$,

$$
\sum_{C \in \mathcal{R} / G}(-1)^{|C|} \mathrm{k}\left(N_{\hat{G}}(C), \hat{B}, d, \rho,[r]\right)=0 .
$$

Similarly, if $Z=1$, then the projective conjecture is the ordinary conjecture. 
Note that if $p=2$ or 3 , then the conjecture is equivalent to Dade's conjecture.

If, moreover, $\hat{E}$ is an extension of $\hat{G}$ centralizing $Z$ and $N_{\hat{E}}(C, \psi)$ is the stabilizer of $\left(N_{\hat{G}}(C), \psi\right)$ in $\hat{E}$, then $N_{\hat{E} / \hat{G}}(C, \psi)=N_{\hat{E}}(C, \psi) / N_{\hat{G}}(C)$ is a subgroup of $\hat{E} / \hat{G}$. For a subgroup $\hat{U} \leq \hat{E} / \hat{G}$, denote by $\mathrm{k}\left(N_{\hat{G}}(C), \hat{B}, d, \hat{U}, \rho,[r]\right)$ the number of characters $\psi$ in $\operatorname{Irr}\left(N_{\hat{G}}(C), \hat{B}, d, \rho,[r]\right)$ such that $N_{\hat{E} / \hat{G}}(C, \psi)=\hat{U}$. In the notation above, Uno's projective invariant conjecture is stated as follows.

Uno's Projective Invariant Conjecture. If $O_{p}(G)=1$ and $\hat{B}$ is a $p$-block of $\hat{G}$ covering $B(\rho)$ with $D(\hat{B}) \neq O_{p}(Z)$, then

$$
\sum_{C \in \mathcal{R} / G}(-1)^{|C|} \mathrm{k}\left(N_{\hat{G}}(C), \hat{B}, d, \hat{U}, \rho,[r]\right)=0 .
$$

In addition, if $\hat{E} / \hat{G}$ is cyclic and $u=|\hat{U}|$, then we set

$$
\mathrm{k}\left(N_{\hat{G}}(C), \hat{B}, d, u, \rho\right)=\mathrm{k}\left(N_{\hat{G}}(C), \hat{B}, d, \hat{U}, \rho\right) .
$$

In particular, if $Z=1$ and $\rho$ is the trivial character of $Z$, then $\hat{G}=G$ and $\hat{B}$ is a block $B$ of $G$; we set $U=\hat{U}$ and

$$
\mathrm{k}(N(C), B, d, U)=\mathrm{k}\left(N_{\hat{G}}(C), \hat{B}, \hat{U}, \rho\right) .
$$

Then the Projective Invariant Conjecture is equivalent to the Invariant Conjecture.

Uno's Invariant Conjecture. If $O_{p}(G)=1$ and $B$ is a $p$-block of $G$ with defect $\mathrm{d}(B)>0$, then

$$
\sum_{C \in \mathcal{R} / G}(-1)^{|C|} \mathrm{k}(N(C), B, d, U,[r])=0 .
$$

Remark 2.1 Suppose $p=5$ and $\hat{G}$ is a covering group of $G=\mathrm{Fi}_{22}$. Then an elementary abelian group $p^{2}$ is a Sylow subgroup of $G$. Let $B \in \operatorname{Blk}(\hat{G})$ with $D(B) \simeq p^{2}$, so that $B$ has p-local rank two (see [2]). Let $R$ be a radical subgroup of $G$ such that $R<D(B)$ and $b \in \operatorname{Blk}\left(N_{G}(R)\right)$ with $b^{G}=B$. Then $b$ has $p$-local rank one or zero, and by [2, Theorem 1.4], Uno's projective conjecture holds for b. In particular,

$$
\mathrm{k}\left(N_{\hat{G}}(R), b, d, \rho,[r]\right)=\mathrm{k}\left(N_{\hat{G}}(D(b)), b, d, \rho,[r]\right)
$$

for any $\rho \in \operatorname{Irr}(Z(\hat{G}))$. Thus Uno's projective conjecture for $B$ is equivalent to the equation

$$
\mathrm{k}(\hat{G}, B, d, \rho,[r])=\mathrm{k}\left(N_{\hat{G}}(D(B)), B, d, \rho,[r]\right) .
$$

In Section 5, we will use the following lemma, whose proof is straightforward.

Lemma 2.2 Let $\sigma: O_{p}(G)<P_{1}<\ldots<P_{m-1}<Q=P_{m}<P_{m+1}<\ldots<P_{\ell}$ be a fixed radical $p$-chain of a finite group $G$, where $1 \leq m<\ell$. Suppose

$$
\sigma^{\prime}: O_{p}(G)<P_{1}<\ldots<P_{m-1}<P_{m+1}<\ldots<P_{\ell}
$$

is also a radical p-chain such that $N_{G}(\sigma)=N_{G}\left(\sigma^{\prime}\right)$. Let $\mathcal{R}^{-}(\sigma, Q)$ be the subfamily of $\mathcal{R}(G)$ consisting of chains $C$ whose $(\ell-1)$-th subchain $C_{\ell-1}$ is conjugate to $\sigma^{\prime}$ in $G$, and $\mathcal{R}^{0}(\sigma, Q)$ the subfamily of $\mathcal{R}(G)$ consisting of chains $C$ whose $\ell$-th subchain $C_{\ell}$ is conjugate to $\sigma$ in $G$. Then the map $g$ sending any $O_{p}(G)<P_{1}<\ldots<P_{m-1}<P_{m+1}<$ $\ldots<P_{\ell}<\ldots$ in $\mathcal{R}^{-}(\sigma, Q)$ to $O_{p}(G)<P_{1}<\ldots<P_{m-1}<Q<P_{m+1}<\ldots<P_{\ell}<\ldots$ induces a bijection, denoted again by $g$, from $\mathcal{R}^{-}(\sigma, Q)$ onto $\mathcal{R}^{0}(\sigma, Q)$. Moreover, for any $C$ in $\mathcal{R}^{-}(\sigma, Q)$, we have $|C|=|g(C)|-1$ and $N_{G}(C)=N_{G}(g(C))$. 


\section{The modified local strategy}

The maximal subgroups of $\mathrm{Fi}_{22}$ were classified by Flaass [14] and Wilson [21]. Using this classification, we know that each radical 2- or 3-subgroup $R$ of $\mathrm{Fi}_{22}$ is radical in one of the nine maximal subgroups $M$ of $\mathrm{Fi}_{22}$ and further that $N_{\mathrm{Fi}_{22}}(R)=N_{M}(R)$.

In [3] and [4], a modified local subgroup strategy was developed to classify the radical subgroups $R$. We review this method here. Suppose $M$ is a subgroup of $G$ such that $N_{M}(R)=N_{G}(R)$.

Step (1). We first consider the case where $M$ is a $p$-local subgroup. Let $Q=O_{p}(M)$, so that $Q \leq R$. Choose a subgroup $X$ of $M$. We explicitly compute the coset action of $M$ on the cosets of $X$ in $M$; we obtain a group $W$ representing this action, a group homomorphism $f$ from $M$ to $W$, and the kernel $K$ of $f$. For a suitable $X$, we have $K=Q$ and the degree of the action of $W$ on the cosets is usually much smaller than that of $M$. We can now directly classify the radical $p$-subgroup classes of $W$, and the preimages in $M$ of the radical subgroup classes of $W$ are the radical subgroup classes of $M$.

Step (2). Now consider the case where $M$ is not $p$-local. We may be able to find its radical $p$-subgroup classes directly. Alternatively, we find a (maximal) subgroup $K$ of $M$ such that $N_{K}(R)=N_{M}(R)$ for each radical subgroup $R$ of $M$. If $K$ is $p$-local, then we apply Step (1) to $K$. If $K$ is not $p$-local, we can replace $M$ by $K$ and repeat Step (2).

Steps (1) and (2) constitute the modified local strategy. After applying the strategy, possible fusions among the resulting list of radical subgroups can be decided readily by testing whether the subgroups in the list are pairwise $\mathrm{Fi}_{22}$-conjugate.

In investigating the conjectures for $\mathrm{Fi}_{22}$, we used the minimal degree representation of $\mathrm{Fi}_{22}$ as a permutation group on 3510 points. Its maximal subgroups were constructed using the details supplied in [9] and the black-box algorithms of Wilson [20]. We also made extensive use of the algorithm described in [10] to construct random elements, and the procedures described in [3] and [4] for deciding the conjectures. We used the minimal degree representation of $\mathrm{Fi}_{22} .2$ as a permutation group on 3510 points, and that of $2 . \mathrm{Fi}_{22}$ as a permutation subgroup of $\mathrm{Fi}_{23}$ on 31671 points. In investigating the projective conjecture for $3 . \mathrm{Fi}_{22}$ and $6 . \mathrm{Fi}_{22}$, we constructed and used representations of 3. $\mathrm{Fi}_{22}$ (and $3 . \mathrm{Fi}_{22} .2$ ) and $6 . \mathrm{Fi}_{22}$ as permutation groups on 185328 and 370656 points, respectively. The representation of $6 . \mathrm{Fi}_{22}$ is available from the ATLAS of Finite Group Representations [6].

The computations reported in this paper were carried out using MAGMA V.2.6-2 on a Sun UltraSPARC Enterprise 4000 server.

\section{Radical subgroups and weights of $\mathrm{Fi}_{22}$}

Let $\Phi(G, p)$ be a set of representatives for conjugacy classes of radical subgroups of $G$. For $H, K \leq G$, we write $H \leq_{G} K$ if $x^{-1} H x \leq K$; and write $H \in_{G} \Phi(G, p)$ if $x^{-1} H x \in \Phi(G, p)$ for some $x \in G$. We shall follow the notation of [9]. In particular, if $p$ is odd, then $p_{+}^{1+2 \gamma}$ is an extra-special group of order $p^{1+2 \gamma}$ with exponent $p$; if $\delta$ is + or - , then $2_{\delta}^{1+2 \gamma}$ is an extra-special group of order $2^{1+2 \gamma}$ with type $\delta$. If $X$ and $Y$ 
are groups, we use $X . Y$ and $X: Y$ to denote an extension and a split extension of $X$ by $Y$, respectively. Given a positive integer $n$, we use $E_{p^{n}}$ or simply $p^{n}$ to denote the elementary abelian group of order $p^{n}, \mathbb{Z}_{n}$ or simply $n$ to denote the cyclic group of order $n$, and $D_{2 n}$ to denote the dihedral group of order $2 n$.

Let $G$ be the simple Fischer group $\mathrm{Fi}_{22}$ and $E=A u t(G)=G .2$. Then

$$
|G|=2^{17} \cdot 3^{9} \cdot 5^{2} \cdot 7 \cdot 11 \cdot 13,
$$

and we may suppose $p \in\{2,3,5\}$, since both conjectures hold for a block with a cyclic defect group by [11, Theorem 7.1] and [2, Theorem 5.1].

We denote by $\operatorname{Irr}^{0}(H)$ the set of ordinary irreducible characters of $p$-defect 0 of a finite group $H$ and by $\mathrm{d}(H)$ the number $\log _{p}(|H|)$. Given $R \in \Phi(G, p)$, let $C(R)=$ $C_{G}(R)$ and $N=N_{G}(R)$. If $B_{0}=B_{0}(G)$ is the principal $p$-block of $G$, then (c.f. (4.1) of $[3])$

$$
\mathcal{W}\left(B_{0}\right)=\sum_{R}\left|\operatorname{Irr}^{0}(N / C(R) R)\right|,
$$

where the summation runs over the subgroups $R$ in $\Phi(G, p)$ for which $\mathrm{d}(C(R) R / R)=$ 0 . The character table of $N / C(R) R$ can be calculated by MAGma, and so we find $\left|\operatorname{Irr}^{0}(N / C(R) R)\right|$.

Proposition 4.1 Let $G=\mathrm{Fi}_{22}$ and $E=\operatorname{Aut}(G)=G .2$. Then the non-trivial radical p-subgroups $R$ of $G$ (up to conjugacy) and their local structures are given in Tables 1 and 2 according as $p \geq 3$ or $p=2$, where $S \in \operatorname{Syl}_{3}(G)$ is a Sylow 3-subgroup, $H^{*}$ denotes a subgroup of $G$ such that $H^{*} \simeq H$ and $H^{*} \neq_{G} H$. If $p=3$, then $\tau$ permutes the pairs $\left(3^{3+3},\left(3^{3+3}\right)^{*}\right)$ and $\left(3_{+}^{1+6} \cdot 3,\left(3_{+}^{1+6} .3\right)^{*}\right)$ for some $\tau \in E \backslash G$. If $p=2$, then $N_{E}(R)=N .2$ for all radical subgroups $R$.

\begin{tabular}{|r|r|r|r|l|}
\hline$R$ & $C(R)$ & $N_{G}(R)$ & $N_{E}(R)$ & $\left|\operatorname{Irr}^{0}(N / C(R) R)\right|$ \\
\hline \hline 5 & $5 \times S_{5}$ & $5: 4 \times S_{5}$ & $2 \times 5: 4 \times S_{5}$ & \\
$5^{2}$ & $5^{2}$ & $5^{2}: 4 S_{4}$ & $2 \times 5^{2}: 4 S_{4}$ & 16 \\
\hline 3 & $3 \times U_{4}(3): 2$ & $S_{3} \times U_{4}(3): 2$ & $N .2$ & \\
$3^{5}$ & $3^{5}$ & $3^{5}: U_{4}(2): 2$ & $N .2$ & 2 \\
$3^{3+3}$ & $3^{3}$ & $3^{3+3}: L_{3}(3)$ & $N$ & 1 \\
$\left(3^{3+3}\right)^{*}$ & $3^{3}$ & $\left(3^{3+3}\right)^{*}: L_{3}(3)$ & $N$ & 1 \\
$3_{+}^{1+6}$ & 3 & $3_{+}^{1+6} .2^{3+4}: 3^{2}: 2$ & $N .2$ & 4 \\
$3_{+}^{1+6} .3$ & 3 & $3_{+}^{1+6} \cdot 3: 2 S_{4}$ & $N$ & 2 \\
$3^{5} .3^{3}$ & 3 & $3^{5} .3^{3} \cdot\left(2 \times S_{4}\right)$ & $N .2$ & 4 \\
$\left(3_{+}^{1+6} .3\right)^{*}$ & 3 & $\left(3_{+}^{1+6} .3\right)^{*} .2 S_{4}$ & $N$ & 2 \\
$3^{5}: 3_{+}^{1+2}$ & $3^{2}$ & $3^{5}: 3_{+}^{1+2} .2 S_{4}$ & $N .2$ & 2 \\
$S$ & 3 & $S .2^{2}$ & $N .2$ & 4 \\
\hline
\end{tabular}

Table 1: Non-trivial radical $p$-subgroups of $\mathrm{Fi}_{22}$ with $p \geq 3$ 
Proof: Case (1). Suppose $p=5$, so that by [9, p. 156], $G=\mathrm{Fi}_{22}$ has a unique class of elements $x$ of order 5 and $C(x)=5 \times S_{5}$ and $N(\langle x\rangle)=5: 4 \times S_{5}$ (see [21, p. 207]). Thus $S \in \operatorname{Syl}_{5}(G)$ is elementary abelian of order $25, N(S)=5^{2}: 4 S_{4}$ and $N_{N(\langle x\rangle)}(S)=5: 4 \times 5: 4$.

Case (2). Suppose $p=3, i \in\{1, \ldots, 4\}$, and $M_{i}$ is a maximal subgroup of $G$ where $M_{1}=N(3 A) \simeq S_{3} \times U_{4}(3): 2, M_{2}=N(3 B) \simeq 3_{+}^{1+6}: 2^{3+4}: 3^{2}: 2, M_{3} \simeq O_{7}(3) \simeq M_{4}$. By [21, p. 203], we may suppose a 3-local subgroup $R$ of $G$ is a subgroup of some $M_{i}$ with $N_{G}(R)=N_{M_{i}}(R)$.

The subgroups $M_{1}$ and $M_{2}$ are normalizers of some $3 A$ and $3 B$ elements, so we can easily construct them in $G$. The generators of a maximal subgroup, say $M_{3} \simeq O_{7}(3)$ are identified by Wilson [6], using standard generators of $\mathrm{Fi}_{22}$. Construct a maximal subgroup $K \simeq 3^{5}: U_{4}(2): 2$ of $M_{3}$ and then use the modified local strategy to obtain all radical subgroups of $K$ (see Case (2.3) below). One of the radical subgroups $R$ of $K$ is isomorphic to $3^{5}: 3_{+}^{1+2}$ with center $Z(R)=3^{2}$. The group $R$ has exactly two conjugacy classes of subgroups, say $Q_{1}$ and $Q_{2}$, satisfying the following conditions: $\left|Q_{1}\right|=\left|Q_{2}\right|=3^{6}, Q_{1} \simeq Q_{2} \simeq 3^{3+3}, N_{M_{3}}\left(Q_{1}\right)=N_{G}\left(Q_{1}\right) \simeq 3^{3+3}: L_{3}(3)$ and $N_{M_{3}}\left(Q_{2}\right) \neq$ $N_{G}\left(Q_{2}\right) \simeq 3^{3+3}: L_{3}(3)$. The group $N_{G}\left(Q_{2}\right)$ is a maximal subgroup of $M_{4} \simeq O_{7}(3)$, and $M_{4}$ can be constructed using $N_{G}\left(Q_{2}\right)$ and some random element of $G$. We then apply the modified local strategy to each $M_{i}$.

Case (2.1) We may take

$$
\Phi\left(M_{2}, 3\right)=\left\{3_{+}^{1+6}, 3_{+}^{1+6} \cdot 3,3^{5} \cdot 3^{3},\left(3_{+}^{1+6} \cdot 3\right)^{*}, S\right\},
$$

and moreover, $N(R)=N_{M_{2}}(R)$ for each $R \in \Phi\left(M_{2}, 3\right)$. We may suppose $\Phi\left(M_{2}, 3\right) \subseteq$ $\Phi(G, 3)$. Since $N_{E}\left(3_{+}^{1+6} \cdot 3\right)=N_{G}\left(3_{+}^{1+6} .3\right)$ and $N_{E}\left(3_{+}^{1+6}\right)=N_{G}\left(3_{+}^{1+6}\right) .2$, it follows that $\tau$ permutes the pair $\left(3_{+}^{1+6} .3,\left(3_{+}^{1+6} .3\right)^{*}\right)$.

Case (2.2) We may take

$$
\Phi\left(M_{1}, 3\right)=\left\{3,3^{5}, 3 \times 3_{+}^{1+4}, S^{\prime}\right\},
$$

and moreover, $N(R) \neq N_{M_{1}}(R)$ for $R \in \Phi\left(M_{1}, 3\right) \backslash\{3\}$ and $N_{E}(R)=N_{G}(R) .2$, where $S^{\prime} \in \operatorname{Syl}_{3}\left(M_{1}\right)$. In addition, $C\left(3^{5}\right)=3^{5}, N\left(3^{5}\right) \simeq S_{3} \times 3^{4}: S_{6}, C\left(3 \times 3_{+}^{1+4}\right)=3^{2}$, $N\left(3 \times 3_{+}^{1+4}\right) \simeq S_{3} \times 3_{+}^{1+4} .2 S_{4}: 2, C\left(S^{\prime}\right)=3^{2}, N\left(S^{\prime}\right) \simeq S_{3} \times 3_{+}^{1+4} .3 . D_{8}$.

Case (2.3) Let $L_{1} \simeq 3_{+}^{1+6}:\left(2 A_{4} \times A_{4}\right) \cdot 2, L_{2} \simeq 3^{3+3}: L_{3}(3)$ and $L_{3}=3^{5}: U_{4}(2): 2$ be the maximal subgroups of $M_{3}=O_{7}(3)$ (cf. [9, p. 109]), so that by the Borel-Tits theorem [8], we may suppose each radical subgroup of $O_{7}(3)$ is a subgroup of $\Phi\left(L_{i}, 3\right)$ for some $i$ with $N(R)=N_{L_{i}}(R)$.

We may take

$$
\Phi\left(L_{1}, 3\right)=\left\{3_{+}^{1+6}, 3^{5}: 3^{3},\left(3_{+}^{1+6} .3\right)^{*}, S\right\},
$$

and moreover, $N(R)=N_{M_{3}}(R)=N_{L_{1}}(R)$ for $R \in \Phi\left(L_{1}, 3\right) \backslash\left\{3_{+}^{1+6}\right\}$.

We may take

$$
\Phi\left(L_{2}, 3\right)=\left\{3^{3+3}, 3_{+}^{1+6} \cdot 3,3^{5}: 3_{+}^{1+2}, S\right\} \subseteq_{G} \Phi(G, 3),
$$

and then $N(R)=N_{M_{3}}(R)$ for all $R \in \Phi\left(L_{2}, 3\right)$. 
We may take

$$
\Phi\left(L_{3}, 3\right)=\left\{3^{5}, 3^{5}: 3^{3}, 3^{5}: 3_{+}^{1+2}, S\right\} \subseteq_{G} \Phi(G, 3),
$$

and so $N(R)=N_{M_{3}}(R)=N_{L_{3}}(R)$ for all $R \in \Phi\left(L_{3}, 3\right)$.

It follows that

$$
\Phi\left(M_{3}, 3\right)=\left\{3^{5}, 3^{3+3}, 3_{+}^{1+6},\left(3_{+}^{1+6} \cdot 3\right)^{*}, 3^{5}: 3^{3}, 3^{5}: 3_{+}^{1+2}, S\right\}
$$

and $N(R)=N_{M_{3}}(R)$ for $R \in \Phi\left(M_{3}, 3\right) \backslash\left\{3_{+}^{1+6}\right\}$.

Case (2.4) Since $M_{3}$ and $M_{4}$ are conjugate in $E$ and $O_{3}\left(M_{2}\right)={ }_{G} O_{3}\left(L_{1}\right)$, it follows that

\begin{tabular}{|c|c|c|c|}
\hline$R$ & $C(R)$ & $N$ & $\mid \operatorname{Irr}^{0}(N / C(R) R)$ \\
\hline 2 & $2 . U_{6}(2)$ & $2 . U_{6}(2)$ & \\
\hline $2^{6}$ & $2^{6}$ & $2^{6}: S_{6}(2)$ & 1 \\
\hline $2^{10}$ & $2^{10}$ & $2^{10}: M_{22}$ & 0 \\
\hline $2 \times 2_{+}^{1+8}$ & $2^{2}$ & $\left(2 \times 2_{+}^{1+8}: U_{4}(2)\right): 2$ & 0 \\
\hline $2^{6} .2^{5}$ & 2 & $2^{6} .2^{5} \cdot S_{6}$ & 1 \\
\hline $2^{10} \cdot 2^{3}$ & $2^{3}$ & $2^{10} \cdot 2^{3} \cdot L_{3}(2)$ & 1 \\
\hline $2^{5+8}$ & $2^{5}$ & $2^{5+8} \cdot\left(S_{3} \times A_{6}\right)$ & 2 \\
\hline $2^{5+8} .2$ & $2^{5}$ & $2^{5+8} \cdot 2 \cdot A_{6}$ & 2 \\
\hline $2^{10} \cdot 2^{4}$ & $2^{2}$ & $2^{10} \cdot 2^{4} \cdot S_{5}$ & 0 \\
\hline $2^{5+8} \cdot 2^{2}$ & $2^{2}$ & $2^{5+8} \cdot 2^{2} \cdot\left(S_{3} \times S_{3}\right)$ & 1 \\
\hline$\left(2 \times 2_{+}^{1+8}\right) \cdot 2.2^{4}$ & $2^{2}$ & $\left(2 \times 2_{+}^{1+8}\right) \cdot 2 \cdot 2^{4} \cdot\left(S_{3} \times S_{3}\right)$ & 1 \\
\hline $2^{10} \cdot 2^{2} \cdot 2^{3}$ & 2 & $2^{10} \cdot 2^{2} \cdot 2^{3} \cdot S_{3}$ & 1 \\
\hline $2^{10} \cdot 2^{2} \cdot 2^{4}$ & $2^{2}$ & $2^{10} \cdot 2^{2} \cdot 2^{4} \cdot S_{3}$ & 1 \\
\hline $2^{5+8} \cdot D_{8}$ & 2 & $2^{5+8} \cdot D_{8} \cdot S_{3}$ & 1 \\
\hline $2^{5+8} \cdot 2^{3}$ & $2^{2}$ & $2^{5+8} \cdot 2^{3} \cdot S_{3}$ & 1 \\
\hline $2^{5+8} \cdot D_{8} \cdot 2$ & 2 & $2^{5+8} \cdot D_{8} \cdot 2$ & 1 \\
\hline
\end{tabular}

$$
\Phi\left(M_{4}, 3\right)=\left\{3^{5},\left(3^{3+3}\right)^{*}, 3_{+}^{1+6}, 3_{+}^{1+6} \cdot 3,3^{5}: 3^{3}, 3^{5}: 3_{+}^{1+2}, S\right\}
$$

Table 2: Non-trivial radical 2-subgroups of $\mathrm{Fi}_{22}$

Case (3) For $1 \leq i \leq 5$, let $M_{i}$ be the maximal subgroups of $G$ such that $M_{1} \simeq$ $2 . U_{6}(2), M_{2} \simeq 2^{10}: M_{22}, M_{3} \simeq 2^{6} . S_{6}(2), M_{4} \simeq\left(2 \times 2_{+}^{1+8}: U_{4}(2)\right): 2$ and $M_{5} \simeq 2^{5+8}:\left(S_{3} \times\right.$ $\left.A_{6}\right)$. If $R$ is a non-trivial radical 2-subgroup of $G$, then by Wilson [21, Proposition 4.4] or Flaass [14], we may suppose $R \in \Phi\left(M_{i}, 2\right)$ such that $N(R)=N_{M_{i}}(R)$ for some $i$.

Case (3.1) We may take

$\Phi\left(M_{4}, 2\right)=\left\{2 \times 2_{+}^{1+8}, 2^{6} \cdot 2^{5}, 2^{10} \cdot 2^{4},\left(2 \times 2_{+}^{1+8}\right) \cdot 2 \cdot 2^{4}, 2^{10} \cdot 2^{2} \cdot 2^{3}, 2^{10} \cdot 2^{2} \cdot 2^{4}, 2^{5+8} \cdot D_{8}, 2^{5+8} \cdot D_{8} \cdot 2\right\}$ and moreover, $N(R)=N_{M_{4}}(R)$ for each $R \in \Phi\left(M_{4}, 2\right)$. 
Case (3.2) Let $K_{1}=\left(2 \times 2_{+}^{1+8}\right): U_{4}(2), K_{2}=2^{10}: L_{3}(4), K_{3}=2^{5+8} \cdot\left(3 \times A_{5}\right) .2$ be maximal subgroups of $M_{1} \simeq 2 . U_{6}(2)$. Then we may suppose each $R \in \Phi\left(M_{1}, 2\right)$ is a subgroup of $\Phi\left(K_{i}, 2\right)$ for some $i$ such that $N_{M_{1}}(R)=N_{K_{i}}(R)$.

We may take

$$
\Phi\left(K_{1}, 2\right)=\left\{2 \times 2_{+}^{1+8}, 2^{10} .2^{4},\left(2 \times 2_{+}^{1+8}\right) \cdot 2.2^{4}, 2^{10} \cdot 2^{2} \cdot 2^{4}\right\}
$$

and so $N_{K_{1}}(R)=N_{M_{1}}(R) \neq N(R)$ for each $R \in \Phi\left(K_{1}, 2\right)$. Moreover, $C(R) \simeq 2^{2}$ and

$$
N_{M_{1}}(R)= \begin{cases}\left(2 \times 2_{+}^{1+8}\right): U_{4}(2) & \text { if } R=2 \times 2_{+}^{1+8}, \\ 2^{10} \cdot 2^{4} \cdot A_{5} & \text { if } R=2^{10} \cdot 2^{4} \\ \left(2 \times 2_{+}^{1+8}\right) \cdot 2 \cdot\left(A_{4} \times A_{4}\right) \cdot 2 & \text { if } R=\left(2 \times 2_{+}^{1+8}\right) \cdot 2.2^{4} \\ 2^{10} \cdot 2^{2} \cdot 2^{4} \cdot 3 & \text { if } R=2^{10} \cdot 2^{2} \cdot 2^{4} .\end{cases}
$$

We may take

$$
\Phi\left(K_{2}, 2\right)=\left\{2^{10}, 2^{10} .2^{4}, 2^{5+8} \cdot 2,2^{10} .2^{2} \cdot 2^{4}\right\}
$$

and moreover, $N_{K_{2}}(R)=N_{M_{1}}(R) \neq N(R)$ for each $R \in \Phi\left(K_{2}, 2\right), C\left(2^{10}\right)=2^{10}$, $C\left(2^{5+8} .2\right)=2^{5}, C\left(2^{10} .2^{4}\right)=2^{2}=C\left(2^{10} .2^{2} .2^{4}\right)$, and $N_{M_{1}}\left(2^{5+8} .2\right)=2^{5+8} .2 A_{5}$.

We may take

$$
\Phi\left(K_{3}, 2\right)=\left\{2^{5+8}, 2^{5+8} .2,\left(2 \times 2_{+}^{1+8}\right) 2.2^{4}, 2^{10} .2^{2} .2^{4}\right\},
$$

and moreover, $N_{K_{3}}(R)=N_{M_{2}}(R) \neq N(R)$ for each $R \in \Phi\left(K_{3}, 2\right)$.

It follows that

$$
\Phi\left(M_{1}, 2\right)=\left\{2,2 \times 2_{+}^{1+8}, 2^{10}, 2^{5+8}, 2^{10} \cdot 2^{4}, 2^{5+8} .2,\left(2 \times 2_{+}^{1+8}\right) \cdot 2.2^{4}, 2^{10} \cdot 2^{2} .2^{4}\right\},
$$

and $N_{M_{1}}(R) \neq N(R)$ for each $R \in \Phi\left(M_{1}, 2\right) \backslash\{2\}$.

Case (3.3) We may take

$$
\Phi\left(M_{2}, 2\right)=\left\{2^{10}, 2^{10} .2^{3}, 2^{10} \cdot 2^{4}, 2^{5+8} \cdot 2,2^{10} \cdot 2^{2} \cdot 2^{3}, 2^{5+8} \cdot 2^{3}, 2^{10} \cdot 2^{2} \cdot 2^{4}, 2^{5+8} \cdot D_{8} \cdot 2\right\},
$$

and moreover, $N(R)=N_{M_{2}}(R)$ for each $R \in \Phi\left(M_{2}, 2\right)$.

Case (3.4) We may take $\Phi\left(M_{5}, 2\right)=\left\{2^{5+8}, 2^{5+8} \cdot 2,2^{5+8} \cdot 2^{2},\left(2 \times 2_{+}^{1+8}\right) \cdot 2 \cdot 2^{4}, 2^{10} \cdot 2^{2} \cdot 2^{4}, 2^{5+8} \cdot 2^{3}, 2^{5+8} \cdot D_{8}, 2^{5+8} \cdot D_{8} \cdot 2\right\}$ and moreover, for $R \in \Phi\left(M_{5}, 2\right), N(R)=N_{M_{5}}(R)$.

Case (3.5) We may take

$$
\Phi\left(M_{3}, 2\right)=\left\{2^{6}, 2^{6} \cdot 2^{5}, 2^{6} \cdot 2^{6}, 2^{6} \cdot 2^{3} \cdot 2^{4}, 2^{6} \cdot 2^{3} \cdot 2^{4} \cdot 2,2^{6} \cdot 2^{5} \cdot 2^{3},\left(2^{6} \cdot 2^{5} \cdot 2^{3}\right)^{*}, S^{\prime}\right\},
$$

where $S^{\prime} \in \operatorname{Syl}_{2}\left(M_{3}\right)$. In addition, $N(R)=N_{M_{3}}(R)$ for $R \in\left\{2^{6}, 2^{6} .2^{5}\right\}$ and $N(R) \neq$ $N_{M_{3}}(R)$ for $R \in \Phi\left(M_{3}, 2\right) \backslash\left\{2^{6}, 2^{6} .2^{5}\right\}$. Moreover,

$$
N_{M_{3}}(R)= \begin{cases}2^{6} \cdot 2^{6} \cdot L_{3}(2) & \text { if } R=2^{6} \cdot 2^{6}, \\ 2^{6} \cdot 2^{3} \cdot 2^{4} \cdot\left(S_{3} \times S_{3}\right) & \text { if } R=2^{6} \cdot 2^{3} \cdot 2^{4}, \\ 2^{6} \cdot 2^{3} \cdot 2^{4} \cdot 2 \cdot S_{3} & \text { if } R=2^{6} \cdot 2^{3} \cdot 2^{4} \cdot 2, \\ 2^{6} \cdot 2^{5} \cdot 2^{3} \cdot S_{3} & \text { if } R=2^{6} \cdot 2^{5} \cdot 2^{3}, \\ \left(2^{6} \cdot 2^{5} \cdot 2^{3}\right)^{*} \cdot S_{3} & \text { if } R=\left(2^{6} \cdot 2^{5} \cdot 2^{3}\right)^{*}, \\ S^{\prime} & \text { if } R=S^{\prime} .\end{cases}
$$


This completes the classification of radical 2-subgroups of $G$. The centralizers and normalizers of $R \in \Phi(G, 2)$ are given by MAGMA.

Lemma 4.2 Suppose $p=2,3$ or 5 , and suppose $G$ is a covering group of $\mathrm{Fi}_{22}$, and $\rho$ a faithful linear character of $Z(G)$. Let $B_{0}=B_{0}(G)$ be the principal block of $G$, $\operatorname{Irr}^{+}(G \mid \rho)$ the set of characters of $\operatorname{Irr}(G)$ with positive $p$-defect and covering $\rho$, and $\operatorname{Blk}^{*}(G, p, \rho)$ the set of $p$-blocks of $G$ with a non-cyclic defect group and covering the block $B(\rho)$. If a defect group $D(B)$ of $B \in \operatorname{Blk}(G)$ is cyclic, then $\operatorname{Irr}(B)$ and $\ell(B)$ are given by [15, p. 218].

(a) If $G=\mathrm{Fi}_{22}$, then $\rho$ is the trivial character, $\operatorname{Blk}^{*}(G, p, \rho)=\left\{B_{0}\right\}, \operatorname{Irr}\left(B_{0}\right)=$ $\operatorname{Irr}^{+}(G \mid \rho) \backslash\left(\cup_{B^{\prime}} \operatorname{Irr}\left(B^{\prime}\right)\right)$ and

$$
\ell\left(B_{0}\right)= \begin{cases}16 & \text { if } p=5 \\ 22 & \text { if } p=3, \\ 14 & \text { if } p=2,\end{cases}
$$

where $B^{\prime}$ runs over the blocks of $G$ with cyclic defect groups.

(b) Suppose $G=2 . \mathrm{Fi}_{22}$, so that $Z(G)$ has a unique faithful linear character $\xi$. If $p=3,5$, then $\operatorname{Blk}^{*}(G, p, \xi)=\left\{B_{1}\right\}$ such that $\operatorname{Irr}\left(B_{1}\right)=\operatorname{Irr}^{+}(G \mid \xi) \backslash\left(\cup_{B^{\prime}} \operatorname{Irr}\left(B^{\prime}\right)\right)$; if $p=2$, then $\operatorname{Blk}^{*}(G, p, \xi)=\left\{B_{0}, B_{1}\right\}$ and in the notation of $[9, \mathrm{p} .156], \operatorname{Irr}\left(B_{1}\right)=$ $\left\{\chi_{63}, \chi_{64}, \chi_{113}, \chi_{114}\right\}, D\left(B_{1}\right) \simeq 2^{2}$ and $\operatorname{Irr}\left(B_{0}\right)=\operatorname{Irr}^{+}(G) \backslash\left(\operatorname{Irr}\left(B_{1}\right) \cup\left(\cup_{B^{\prime}} \operatorname{Irr}\left(B^{\prime}\right)\right)\right)$, where $\operatorname{Irr}^{+}(G)$ is the character in $\operatorname{Irr}(G)$ with positive defect and $B^{\prime}$ runs over the blocks of $G$ with cyclic defect groups.

(c) Suppose $G=3 . \mathrm{Fi}_{22}$, so that $Z(G)$ has two faithful linear characters $\zeta_{1}$ and $\zeta_{2}$. If $p=2,5$, then $\operatorname{Blk}^{*}\left(G, p, \zeta_{i}\right)=\left\{B_{i}\right\}$, and $\operatorname{Irr}\left(B_{i}\right)=\operatorname{Irr}^{+}\left(G \mid \zeta_{i}\right) \backslash\left(\cup_{B^{\prime}} \operatorname{Irr}\left(B^{\prime}\right)\right)$; if $p=3$, then $\operatorname{Blk}^{*}\left(G, p, \zeta_{i}\right)=\left\{B_{0}, B_{i}\right\}$ and in the notation of $[9$, p. 156], we may suppose

$$
\operatorname{Irr}\left(B_{i}\right)= \begin{cases}\left\{\chi_{j}: j \in\{29,38,49,98,99,114,115,126,127\}\right\} & \text { if } i=1, \\ \left\{\chi_{j}: j \in\{48,58,65,124,125,156,157,162,163\}\right\} & \text { if } i=2,\end{cases}
$$

and $\operatorname{Irr}\left(B_{0}\right)=\operatorname{Irr}^{+}(G) \backslash\left(\operatorname{Irr}\left(B_{1}\right) \cup \operatorname{Irr}\left(B_{2}\right) \cup\left(\cup_{B^{\prime}} \operatorname{Irr}\left(B^{\prime}\right)\right)\right)$, where $B^{\prime}$ runs over the blocks of $G$ with cyclic defect groups.

(d) Suppose $G=6 . \mathrm{Fi}_{22}$, so that $Z(G)$ has two faithful linear characters $\eta_{1}$ and $\eta_{2}$. If $p=2,5$, then $\operatorname{Blk}^{*}\left(G, p, \eta_{i}\right)=\left\{B_{i}\right\}$ and $\operatorname{Irr}\left(B_{i}\right)=\operatorname{Irr}^{+}\left(G \mid \eta_{i}\right) \backslash\left(\cup_{B^{\prime}} \operatorname{Irr}\left(B^{\prime}\right)\right)$; if $p=3$, then $\operatorname{Blk}^{*}\left(G, p, \eta_{i}\right)=\left\{B_{1}\right\}$ such that

$$
\left(\operatorname{Irr}^{+}\left(G \mid \eta_{i}\right) \backslash\left(\cup_{B^{\prime}} \operatorname{Irr}\left(B^{\prime}\right)\right)\right) \subseteq \operatorname{Irr}\left(B_{1}\right)
$$

for $i=1,2$, where $B^{\prime}$ runs over the blocks of $G$ with cyclic defect groups.

Proof: If $B \in \operatorname{Blk}^{+}(G, p)$ is non-principal with $D=D(B)$, then $\operatorname{Irr}^{0}(C(D) D / D)$ has a non-trivial character $\theta$ and $N(\theta) / C(D) D$ is a $p^{\prime}$-group, where $N(\theta)$ is the stabilizer 
of $\theta$ in $N(D)$. By Proposition 4.1, $D \in_{G}\{5,3,2\}$ and $D$ is cyclic. In particular, $\operatorname{Irr}(B)$ and $\ell(B)$ are given by $[15, \mathrm{p} .218]$.

If $\ell_{p}(G)$ is the number of $p$-regular $G$-conjugacy classes, then $\ell\left(B_{0}\right)$ can be calculated by the following equation due to Brauer:

$$
\ell_{p}(G)=\bigcup_{B \in \operatorname{Blk}^{+}(G, p)} \ell(B)+\left|\operatorname{Irr}^{0}(G)\right|
$$

Suppose $B \in \operatorname{Blk}^{*}(G, p, \rho)$. Using central characters one can show $\operatorname{Irr}(B)$ has the indicated description.

Theorem 4.3 Let $B$ be a p-block of $G=\mathrm{Fi}_{22}$ with a non-cyclic defect group. Then $\mathcal{W}(B)=\ell(B)$.

Proof: By Lemma 4.2, $B=B_{0}$ and the theorem follows by Proposition 4.1, Lemma 4.2 and (4.1).

\section{$5 \quad$ Radical chains of $\mathrm{Fi}_{22}$}

Let $G=\mathrm{Fi}_{22}, C \in \mathcal{R}(G)$ and $N(C)=N_{G}(C)$. In this section, we do some cancellations in the alternating sum of Uno's conjecture. First we list some radical $p$-chains $C(i)$ with their normalizers, then reduce the proof of the conjecture to the subfamily $\mathcal{R}^{0}(G)$ of $\mathcal{R}(G)$ consisting of the union of $G$-orbits of all $C(i)$. The subgroups of the $p$-chains in Tables 3 and 4 are given either by Tables 1 and 2 or in the proofs of Proposition 4.1 and Lemma 5.1.

Lemma 5.1 Let $G=\mathrm{Fi}_{22}, E=G .2$ and let $\mathcal{R}^{0}(G)$ be the $G$-invariant subfamily of $\mathcal{R}(G)$ such that

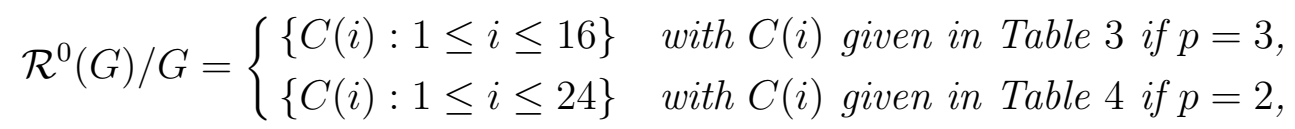

Then

$$
\sum_{C \in \mathcal{R}(G) / G}(-1)^{|C|} \mathrm{k}(N(C), B, d,[r])=\sum_{C \in \mathcal{R}^{0}(G) / G}(-1)^{|C|} \mathrm{k}(N(C), B, d,[r])
$$

for all integers $d, r \geq 0$. If $p=3$, then an element $\tau \in E \backslash G$ permutes the pairs $(C(i), C(j))$ for $(i, j) \in\{(7,9),(8,10),(11,15),(14,16)\}$. If $p=2$, then $N_{E}(C(i))=$ $N(C(i)) .2$ for each $i$.

Proof: Suppose $C^{\prime}$ is a radical chain such that

$$
C^{\prime}: 1<P_{1}^{\prime}<\ldots<P_{m}^{\prime}
$$




\begin{tabular}{|c|c|c|c|}
\hline$C$ & & $N(C)$ & $N_{E}(C)$ \\
\hline$C(1)$ & 1 & $\mathrm{Fi}_{22}$ & $\mathrm{Fi}_{22} .2$ \\
\hline$C(2)$ & $1<5$ & $5: 4 \times S_{5}$ & $N(C) \times 2$ \\
\hline$C(3)$ & $1<5<5^{2}$ & $5: 4 \times 5: 4$ & $N(C) \times 2$ \\
\hline$C(4)$ & $1<5^{2}$ & $5^{2}: 4 S_{4}$ & $N(C) \times 2$ \\
\hline$C(1)$ & 1 & $\mathrm{Fi}_{22}$ & $\mathrm{Fi}_{22 .} \cdot 2$ \\
\hline$C(2)$ & $1<3$ & $S_{3} \times U_{4}(3): 2$ & $N(C) .2$ \\
\hline$C(3)$ & $1<3<3^{5}$ & $S_{3} \times 3^{4}: S_{6}$ & $N(C) .2$ \\
\hline$C(4)$ & $1<3<3^{5}<S^{\prime}$ & $S_{3} \times 3_{+}^{1+4} \cdot 3 . D_{8}$ & $N(C) .2$ \\
\hline$C(5)$ & $1<3<3 \times 3_{+}^{1+4}$ & $S_{3} \times 3_{+}^{1+4} \cdot 2 S_{4}: 2$ & $N(C) .2$ \\
\hline$C(6)$ & $1<3^{5}$ & $3^{5}: U_{4}(2): 2$ & $N(C) .2$ \\
\hline$C(7)$ & $1<3^{3+3}<3^{5}: 3_{+}^{1+2}$ & $3^{5}: 3_{+}^{1+2}: 2 S_{4}$ & $N(C)$ \\
\hline$C(8)$ & $1<3^{3+3}$ & $3^{3+3}: L_{3}(3)$ & $N(C)$ \\
\hline$C(9)$ & $1<\left(3^{3+3}\right)^{*}<3^{5}: 3_{+}^{1+2}$ & $3^{5}: 3_{+}^{1+2}: 2 S_{4}$ & $N(C)$ \\
\hline$C(10)$ & $1<\left(3^{3+3}\right)^{*}$ & $\left(3^{3+3}\right)^{*}: L_{3}(3)$ & $N(C)$ \\
\hline$C(11)$ & $1<3_{+}^{1+6}<3_{+}^{1+6} .3$ & $3_{+}^{1+6} \cdot 3.2 S_{4}$ & $N(C)$ \\
\hline$C(12)$ & $1<3_{+}^{1+6}$ & $3_{+}^{1+6} \cdot 2^{3+4}: 3^{2}: 2$ & $N(C) .2$ \\
\hline$C(13)$ & $1<3_{+}^{1+6}<3^{5}: 3^{3}$ & $3^{5} \cdot 3^{3}:\left(2 \times S_{4}\right)$ & $N(C) .2$ \\
\hline$C(14)$ & $1<3_{+}^{1+6}<3_{+}^{1+6} .3<S$ & $S .2^{2}$ & $N(C)$ \\
\hline$C(15)$ & $1<3_{+}^{1+6}<\left(3_{+}^{1+6}: 3\right)^{*}$ & $\left(3_{+}^{1+6}: 3\right)^{*}: 2 S_{4}$ & $N(C)$ \\
\hline$C(16)$ & $1<3_{+}^{1+6}<\left(3_{+}^{1+6} .3\right)^{*}<S$ & $S .2^{2}$ & $N(C)$ \\
\hline
\end{tabular}

Table 3: Some radical $p$-chains of $\mathrm{Fi}_{22}$ with $p$ odd

Let $C \in \mathcal{R}(G)$ be given by $(2.1)$ with $P_{1} \in \Phi(G, 3)$.

Case (1) Suppose $p=3$, and $R \in \Phi\left(L_{3}, 3\right) \backslash\left\{3^{5}\right\}$ given by (4.6). Let $\sigma(R): 1<Q=$ $3^{5}<R$, so that $\sigma(R)^{\prime}: 1<R$. Then $\sigma(R)$ and $\sigma(R)^{\prime}$ satisfy the conditions of Lemma 2.2 , so there is a bijection $g$ from $\mathcal{R}^{-}\left(\sigma(R), 3^{5}\right)$ onto $\mathcal{R}^{0}\left(\sigma(R)^{\prime}, 3^{5}\right)$ such that $N\left(C^{\prime}\right)=$ $N\left(g\left(C^{\prime}\right)\right), N_{E}\left(C^{\prime}\right)=N_{E}\left(g\left(C^{\prime}\right)\right)$ and $\left|C^{\prime}\right|=\left|g\left(C^{\prime}\right)\right|-1$ for each $C^{\prime} \in \mathcal{R}^{-}\left(\sigma(R), 3^{5}\right)$. Thus

$$
\mathrm{k}\left(N\left(C^{\prime}\right), B, d, u,[r]\right)=\mathrm{k}\left(N\left(g\left(C^{\prime}\right)\right), B, d, u,[r]\right),
$$

and we may suppose

$$
C \notin \underset{R \in \Phi\left(L_{3}, 3\right) \backslash\left\{3^{5}\right\}}{ }\left(\mathcal{R}^{-}\left(\sigma(R), 3^{5}\right) \cup \mathcal{R}^{0}\left(\sigma(R), 3^{5}\right)\right) .
$$

Thus $P_{1} \notin\left\{3^{5}: 3^{3}, 3^{5}: 3_{+}^{1+2}, S\right\}$, and if $P_{1}=3^{5}$ then $C={ }_{G} C(6)$. We may suppose

$$
P_{1} \in\left\{3,3^{3+3},\left(3^{3+3}\right)^{*}, 3_{+}^{1+6}, 3_{+}^{1+6} .3,\left(3_{+}^{1+6} .3\right)^{*}\right\} \subseteq \Phi(G, 3) .
$$

Let $C^{\prime}: 1<3<S^{\prime}$ and $g\left(C^{\prime}\right): 1<3<3 \times 3_{+}^{1+4}<S^{\prime}$, where $S^{\prime}, 3 \times 3_{+}^{1+4} \in \Phi\left(M_{1}, 3\right)$. Then $N\left(C^{\prime}\right)=N\left(g\left(C^{\prime}\right)\right)$ and $N_{E}\left(C^{\prime}\right)=N_{E}\left(g\left(C^{\prime}\right)\right)=S^{\prime} .2$ and we may suppose $C \neq_{G}$ 
$C^{\prime}, g\left(C^{\prime}\right)$. Since $\Phi\left(S_{3} \times 3^{4}: S_{6}, 3\right)=\left\{3^{5}, S^{\prime}\right\} \subseteq_{G} \Phi\left(M_{1}, 3\right)$, it follows that if $P_{1}=3$, then $C \in_{G}\{C(2), C(3), C(4), C(5)\}$.

By $(4.5), \Phi\left(\left(3^{3+3}\right)^{*}: L_{3}(3), 3\right)=\left\{\left(3^{3+3}\right)^{*}, 3_{+}^{1+6} \cdot 3,3^{5}: 3_{+}^{1+2}, S\right\}$. Let $\sigma: 1<Q=$ $\left(3^{3+3}\right)^{*}<3_{+}^{1+6} .3$, so that $\sigma^{\prime}: 1<3_{+}^{1+6}$.3. A similar proof to above shows that there is a bijection $g$ from $\mathcal{R}^{-}\left(\sigma,\left(3^{3+3}\right)^{*}\right)$ onto $\mathcal{R}^{0}\left(\sigma,\left(3^{3+3}\right)^{*}\right)$ such that $N_{E}\left(C^{\prime}\right)=N\left(C^{\prime}\right)=$ $N\left(g\left(C^{\prime}\right)\right)=N_{E}\left(g\left(C^{\prime}\right)\right)$ and $\left|C^{\prime}\right|=\left|g\left(C^{\prime}\right)\right|-1$ for each $C^{\prime} \in \mathcal{R}^{-}\left(\sigma,\left(3^{3+3}\right)^{*}\right)$, so that (5.3) holds. We may suppose $P_{1} \neq_{G} 3_{+}^{1+6} .3$ and if $P_{1}={ }_{G}\left(3^{3+3}\right)^{*}$, then $P_{2} \in_{G}\left\{3^{5}: 3_{+}^{1+2}, S\right\}$.

Let $C^{\prime}: 1<\left(3^{3+3}\right)^{*}<S$ and $g\left(C^{\prime}\right): 1<\left(3^{3+3}\right)^{*}<3^{5}: 3_{+}^{1+2}<S$. Then $N\left(C^{\prime}\right)=$ $N\left(g\left(C^{\prime}\right)\right), N_{E}\left(C^{\prime}\right)=N_{E}\left(g\left(C^{\prime}\right)\right)$ and we may suppose $C \neq_{G} C^{\prime}, g\left(C^{\prime}\right)$. Thus if $P_{1}=$ $\left(3^{3+3}\right)^{*}$, then $C \in_{G}\{C(9), C(10)\}$.

Let $\sigma: 1<Q=3^{3+3}<\left(3_{+}^{1+6} .3\right)^{*}$, so that $\sigma^{\prime}: 1<\left(3_{+}^{1+6} .3\right)^{*}$. A similar proof to above shows that we may suppose

$$
C \notin\left(\mathcal{R}^{-}\left(\sigma, 3^{3+3}\right) \cup \mathcal{R}^{0}\left(\sigma, 3^{3+3}\right)\right) .
$$

In particular, we may suppose $P_{1} \neq_{G}\left(3_{+}^{1+6} .3\right)^{*}$ and moreover, if $P_{1}=3^{3+3}$, then $P_{2} \neq \neq_{G}\left(3_{+}^{1+6} .3\right)^{*}$.

Let $C^{\prime}: 1<3^{3+3}<S$ and $g\left(C^{\prime}\right): 1<3^{3+3}<3^{5}: 3_{+}^{1+2}<S$. Then we may suppose $C \neq_{G} C^{\prime}, g\left(C^{\prime}\right)$. So if $P_{1}=3^{3+3}$, then $C \in_{G}\{C(7), C(8)\}$.

Let $C^{\prime}: 1<3_{+}^{1+6}<S$ and $g\left(C^{\prime}\right): 1<3_{+}^{1+6}<3^{5}: 3^{3}<S$. Then $N\left(C^{\prime}\right)=N\left(g\left(C^{\prime}\right)\right)$ and $N_{E}(C)=N_{E}\left(g\left(C^{\prime}\right)\right)=N\left(C^{\prime}\right) .2$, so that (5.3) holds. Thus if $P_{1}=3_{+}^{1+6}$, then $C \in_{G}\{C(i): 11 \leq i \leq 16\}$.

Case (2) Let $R \in \Phi\left(M_{2}, 2\right) \backslash\left\{2^{10}\right\}$, and $\sigma(R): 1<Q=2^{10}<R$, so that $\sigma(R): 1<$ $R$. A similar proof to that of Case (1) shows that we may suppose

$$
C \notin \bigcup_{R \in \Phi\left(M_{2}, 2\right) \backslash\left\{2^{10}\right\}}\left(\mathcal{R}^{-}\left(\sigma(R), 2^{10}\right) \cup \mathcal{R}^{0}\left(\sigma(R), 2^{10}\right)\right) .
$$

Thus if $P_{1}=2^{10}$, then $C={ }_{G} C(14)$. We may assume

$$
P_{1} \in\left\{2,2^{6}, 2 \times 2^{1+8}, 2^{6} \cdot 2^{5}, 2^{5+8}, 2^{5+8} \cdot 2^{2},\left(2 \times 2_{+}^{1+8}\right) \cdot 2.2^{4}, 2^{5+8} \cdot D_{8}\right\} .
$$

Case (2.1) Let

$$
\Omega=\Phi\left(K_{1}, 2\right) \backslash\left\{2 \times 2_{+}^{1+8}\right\}=\left\{2^{10} .2^{4},\left(2 \times 2_{+}^{1+8}\right) \cdot 2.2^{4}, 2^{10} .2^{2} .2^{4}\right\} \subseteq \Phi\left(M_{1}, 2\right),
$$

$R \in \Omega$, and let $\sigma(R): 1<2<Q=2 \times 2_{+}^{1+8}<R$, so that $\sigma(R)^{\prime}: 1<2<R$. A similar proof to Case (1) shows that we may suppose (5.4) holds with $2^{10}$ replaced by $2 \times 2_{+}^{1+8}$ and $\Phi\left(M_{2}, 2\right) \backslash\left\{2^{10}\right\}$ by $\Omega$.

Let $2^{5+8} .2 \in \Phi\left(K_{2}, 2\right)$ and let $\sigma: 1<2<Q=2^{10}<2^{5+8} .2$, so that $\sigma^{\prime}: 1<$ $2<2^{5+8} .2$. Since $N_{N_{E}(2)}\left(2^{5+8} .2\right) \leq N_{N_{E}(2)}\left(2^{10}\right)$, it follows that we may suppose $C \notin$ $\mathcal{R}^{-}\left(\sigma, 2^{10}\right) \cup \mathcal{R}^{0}\left(\sigma, 2^{10}\right)$.

Let $C^{\prime}: 1<2<2^{10}<2^{10} .2^{2} .2^{4}, g\left(C^{\prime}\right): 1<2<2^{10}<2^{10} .2^{4}<2^{10} .2^{2} .2^{4}$, $C^{\prime \prime}: 1<2<2^{5+8}<2^{10} \cdot 2^{2} .2^{4}$ and $g\left(C^{\prime \prime}\right): 1<2<2^{5+8}<\left(2 \times 2_{+}^{1+8}\right) .2 .2^{4}<2^{10} .2^{2} .2^{4}$. Then $N\left(C^{\prime}\right)=N\left(g\left(C^{\prime}\right)\right), N_{E}\left(C^{\prime}\right)=N_{E}\left(g\left(C^{\prime}\right)\right)=N\left(C^{\prime}\right) .2, N\left(C^{\prime \prime}\right)=N\left(g\left(C^{\prime \prime}\right)\right)$ and $N_{E}\left(C^{\prime \prime}\right)=N_{E}\left(g\left(C^{\prime \prime}\right)\right)=N\left(C^{\prime \prime}\right) .2$, so that we may suppose $C \notin\left\{C^{\prime}, g\left(C^{\prime}\right), C^{\prime \prime}, g\left(C^{\prime \prime}\right)\right\}$. It follows that if $P_{1}=2$, then $C \in_{G}\{C(j): 2 \leq j \leq 9\}$. 


\begin{tabular}{|c|c|c|}
\hline$C$ & & $N(C)$ \\
\hline$C(1)$ & 1 & $\mathrm{Fi}_{22}$ \\
\hline$C(2)$ & $1<2$ & $2 . U_{6}(2)$ \\
\hline$C(3)$ & $1<2<2 \times 2_{+}^{1+8}$ & $\left(2 \times 2_{+}^{1+8}\right): U_{4}(2)$ \\
\hline$C(4)$ & $1<2<2^{10}<2^{10} .2^{4}$ & $2^{10} \cdot 2^{4} \cdot A_{5}$ \\
\hline$C(5)$ & $1<2<2^{10}$ & $2^{10}: L_{3}(4)$ \\
\hline$C(6)$ & $1<2<2^{5+8}<2^{5+8} .2$ & $2^{5+8} \cdot 2 \cdot A_{5}$ \\
\hline$C(7)$ & $1<2<2^{5+8}$ & $2^{5+8} \cdot\left(3 \times A_{5}\right) \cdot 2$ \\
\hline$C(8)$ & $1<2<2^{5+8}<\left(2 \times 2_{+}^{1+8}\right) \cdot 2.2^{4}$ & $\left(2 \times 2_{+}^{1+8}\right) \cdot 2 \cdot\left(A_{4} \times A_{4}\right) \cdot 2$ \\
\hline$C(9)$ & $1<2<2^{5+8}<2^{5+8} .2<2^{10} \cdot 2^{2} .2^{4}$ & $2^{10} \cdot 2^{2} \cdot 2^{4} .3$ \\
\hline$C(10)$ & $1<2^{6}$ & $2^{6} . S_{6}(2)$ \\
\hline$C(11)$ & $1<2^{6}<2^{6} .2^{6}$ & $2^{6} .2^{6} \cdot L_{3}(2)$ \\
\hline$C(12)$ & $1<2^{6}<2^{6} .2^{3} .2^{4}<2^{6} .2^{3} .2^{4} .2$ & $2^{6} .2^{3} \cdot 2^{4} \cdot 2 . S_{3}$ \\
\hline$C(13)$ & $1<2^{6}<2^{6} .2^{3} .2^{4}$ & $2^{6} .2^{3} .2^{4}\left(S_{3} \times S_{3}\right)$ \\
\hline$C(14)$ & $1<2^{10}$ & $2^{10}: M_{22}$ \\
\hline$C(15)$ & $1<2 \times 2_{+}^{1+8}<2^{6} .2^{5}$ & $2^{6} \cdot 2^{5} \cdot S_{6}$ \\
\hline$C(16)$ & $1<2 \times 2_{+}^{1+8}$ & $\left(2 \times 2_{+}^{1+8}: U_{4}(2)\right): 2$ \\
\hline$C(17)$ & $1<2 \times 2_{+}^{1+8}<2^{10} .2^{4}$ & $2^{10} \cdot 2^{4} \cdot S_{5}$ \\
\hline$C(18)$ & $1<2 \times 2_{+}^{1+8}<2^{6} .2^{5}<2^{6} .2^{5} .2^{3}$ & $2^{6} \cdot 2^{5} \cdot 2^{3} \cdot S_{3}$ \\
\hline$C(19)$ & $1<2 \times 2_{+}^{1+8}<2^{6} .2^{5}<2^{6} .2^{5} .2^{3}<2^{6} .2^{5} .2^{3} .2$ & $2^{6} \cdot 2^{5} \cdot 2^{3} \cdot 2$ \\
\hline$C(20)$ & $1<2 \times 2_{+}^{1+8}<2^{6} .2^{5}<\left(2^{6} .2^{5} .2^{3}\right)^{*}$ & $\left(2^{6} \cdot 2^{5} \cdot 2^{3}\right)^{*} \cdot S_{3}$ \\
\hline$C(21)$ & $1<2^{5+8}<2^{5+8} .2$ & $2^{5+8} \cdot 2 A_{6}$ \\
\hline$C(22)$ & $1<2^{5+8}$ & $2^{5+8} \cdot\left(S_{3} \times A_{6}\right)$ \\
\hline$C(23)$ & $1<2^{5+8}<\left(2 \times 2_{+}^{1+8}\right) \cdot 2 \cdot 2^{4}$ & $\left(2 \times 2_{+}^{1+8}\right) \cdot 2 \cdot 2^{4} \cdot\left(S_{3} \times S_{3}\right)$ \\
\hline$C(24)$ & $1<2^{5+8}<\left(2 \times 2_{+}^{1+8}\right) \cdot 2.2^{4}<2^{10} \cdot 2^{2} .2^{4}$ & $2^{10} \cdot 2^{2} \cdot 2^{4} \cdot S_{3}$ \\
\hline
\end{tabular}

Table 4: Some radical 2-chains of $\mathrm{Fi}_{22}$

Case (2.2) If $2^{6} .2^{6} \in \Phi\left(M_{3}, 2\right)$, then $N_{M_{3}}\left(2^{6} .2^{6}\right)=2^{6} .2^{6} . L_{3}(2)$ and $N_{M_{3} .2}\left(2^{6} .2^{6}\right)=$ $N_{E}\left(2^{6} \cdot 2^{6}\right)=N\left(2^{6} \cdot 2^{6}\right) \cdot 2$. We may take

$$
\Phi\left(2^{6} \cdot 2^{6} \cdot L_{3}(2), 2\right)=\left\{2^{6} \cdot 2^{6}, 2^{6} \cdot 2^{3} \cdot 3^{4} \cdot 2,2^{6} \cdot 2^{5} \cdot 2^{3}, S^{\prime}\right\} \subseteq \Phi\left(M_{3}, 2\right),
$$

and $N_{2^{6} .2^{6} . L_{3}(2)}(R)=N_{M_{3}}(R), N_{M_{3} .2}(R)=N_{M_{3}}(R) .2 \leq N_{M_{3} .2}\left(2^{6} .2^{6}\right)$ for each $R \in$ $\Phi\left(2^{6} \cdot 2^{6} \cdot L_{3}(2), 2\right)$.

Let $2^{6} .2^{5} \in \Phi\left(M_{3}, 2\right)$, and $\sigma: 1<Q=2^{6}<2^{6} .2^{5}$, so that $\sigma^{\prime}: 1<2^{6} .2^{5}$. A similar proof to Case (1) shows that we may suppose $C \notin \mathcal{R}^{-}\left(\sigma, 2^{6}\right) \cup \mathcal{R}^{0}\left(\sigma, 2^{6}\right)$, since $N\left(2^{6} .2^{5}\right)=N_{M_{3}}\left(2^{6} .2^{5}\right)=2^{6} .2^{5} . S_{6}$ and $N_{E}\left(2^{6} .2^{5}\right)=N_{M_{3} .2^{2}}\left(2^{6} .2^{5}\right)=N\left(2^{6} .2^{5}\right) .2$. In particular, we may suppose $P_{1} \neq \neq_{G} 2^{6} .2^{5}$.

Let $R \in \Phi\left(2^{6} .2^{6} . L_{3}(2), 2\right) \backslash\left\{2^{6} .2^{6}\right\}$, and $\sigma(R): 1<2^{6}<Q=2^{6} .2^{6}<R$, so that $\sigma(R)^{\prime}: 1<2^{6}<R$. Then we may suppose $C \notin \mathcal{R}^{-}\left(\sigma(R), 2^{6} .2^{6}\right) \cup \mathcal{R}^{0}\left(\sigma(R), 2^{6} .2^{6}\right)$. 
Similarly, for $2^{6} .2^{3} .2^{4} \in \Phi\left(M_{3}, 2\right), N_{M_{3}}\left(2^{6} .2^{3} .2^{4}\right)=2^{6} .2^{3} .2^{4} .\left(S_{3} \times S_{3}\right)$ and we may take

$$
\Phi\left(2^{6} \cdot 2^{3} \cdot 2^{4} \cdot\left(S_{3} \times S_{3}\right), 2\right)=\left\{2^{6} \cdot 2^{3} \cdot 2^{4}, 2^{6} \cdot 2^{3} \cdot 2^{4} \cdot 2,\left(2^{6} \cdot 2^{5} \cdot 2^{3}\right)^{*}, S^{\prime}\right\} \subseteq \Phi\left(M_{3}, 2\right) ;
$$

in addition, $N_{2^{6} \cdot 2^{3} \cdot 2^{4} \cdot\left(S_{3} \times S_{3}\right)}(R)=N_{M_{3}}(R)$ and

$$
N_{M_{3} .2}(R)=N_{M_{3}}(R) .2 \leq N_{M_{3} .2}\left(2^{6} \cdot 2^{3} \cdot 2^{4}\right)
$$

for each $R \in \Phi\left(2^{6} .2^{3} .2^{4} .\left(S_{3} \times S_{3}\right), 2\right)$.

Let $\sigma: 1<2^{6}<Q=2^{6} .2^{3} .2^{4}<\left(2^{6} .2^{5} .2^{3}\right)^{*}$, so that $\sigma^{\prime}: 1<2^{6}<\left(2^{6} .2^{5} .2^{3}\right)^{*}$. Then we may suppose $C \notin \mathcal{R}^{-}\left(\sigma, 2^{6} .2^{3} .2^{4}\right) \cup \mathcal{R}^{0}\left(\sigma, 2^{6} .2^{3} .2^{4}\right)$.

Let $C^{\prime}: 1<2^{6}<2^{6} .2^{3} .2^{4}<S^{\prime}$ and $g\left(C^{\prime}\right): 1<2^{6}<2^{6} .2^{3} .2^{4}<2^{6} .2^{3} .2^{4} .2<S^{\prime}$. Then $N\left(C^{\prime}\right)=N\left(g\left(C^{\prime}\right)\right), N_{E}\left(C^{\prime}\right)=N_{E}\left(g\left(C^{\prime}\right)\right)=S^{\prime} .2$ and we may suppose $C \notin_{G}$ $\left\{C^{\prime}, g\left(C^{\prime}\right)\right\}$. It follows that if $P_{1}=2^{6}$, then $C \in_{G}\{C(10), C(11), C(12), C(13)\}$.

Case (2.3) Let $R \in\left\{\left(2 \times 2_{+}^{1+8}\right) \cdot 2.2^{4}, 2^{5+8} . D_{8}\right\} \subseteq \Phi\left(M_{4}, 2\right)$, and $\sigma(R): 1<Q=$ $2 \times 2_{+}^{1+8}<R$, so that $\sigma(R)^{\prime}: 1<R$. Since $N(R)=N_{M_{4}}(R)$ and $N_{E}(R)=N_{M_{4} .2}(R)=$ $N(R) .2$, it follows that we may suppose $C \notin \mathcal{R}^{-}\left(\sigma(R), 2 \times 2_{+}^{1+8}\right) \cup \mathcal{R}^{0}\left(\sigma(R), 2 \times 2_{+}^{1+8}\right)$. In particular, $P_{1} \neq_{G}\left\{\left(2 \times 2_{+}^{1+8}\right) \cdot 2.2^{4}, 2^{5+8} . D_{8}\right\}$ and if $P_{1}=2 \times 2_{+}^{1+8}$ with $|C| \geq 2$, then $P_{2} \in \Phi\left(\left(2 \times 2_{+}^{1+8}: U_{4}(2)\right): 2,2\right) \backslash\left\{2 \times 2_{+}^{1+8},\left(2 \times 2_{+}^{1+8}\right) \cdot 2.2^{4}, 2^{5+8} . D_{8}\right\}$.

Let $2^{10} .2^{4} \in \Phi\left(M_{4}, 2\right)$, so that $N_{M_{4}}\left(2^{10} .2^{4}\right)=N\left(2^{10} .2^{4}\right)=2^{10} .2^{4} . S_{5}$ and $N_{E}\left(2^{10} .2^{4}\right)=$ $N_{M_{4} \cdot 2}\left(2^{10} \cdot 2^{4}\right)=N\left(2^{10} \cdot 2^{4}\right) \cdot 2$. We may take

$$
\Phi\left(2^{10} \cdot 2^{4} \cdot S_{5}, 2\right)=\left\{2^{10} \cdot 2^{4}, 2^{10} \cdot 2^{2} \cdot 2^{3}, 2^{10} \cdot 2^{2} \cdot 2^{4}, S\right\}
$$

and in addition, $N_{2^{10} .2^{4} \cdot S_{5}}(R)=N_{M_{4}}(R)$ and

$$
N_{M_{4} .2}(R)=N_{M_{4}}(R) .2 \leq N_{M_{4} \cdot 2}\left(2^{10} .2^{4}\right)
$$

for each $R \in \Phi\left(2^{10} \cdot 2^{4} \cdot S_{5}, 2\right)$.

Let $R \in \Phi\left(2^{10} .2^{4} . S_{5}, 2\right) \backslash\left\{2^{10} .2^{4}\right\}$, and $\sigma(R): 1<2 \times 2_{+}^{1+8}<Q=2^{10} .2^{4}<$ $R$, so that $\sigma(R)^{\prime}: 1<2 \times 2_{+}^{1+8}<R$. A similar proof to above shows that we may suppose $C \notin \mathcal{R}^{-}\left(\sigma(R), 2^{10} .2^{4}\right) \cup \mathcal{R}^{0}\left(\sigma(R), 2^{10} .2^{4}\right)$. Thus we may suppose $P_{2} \notin$ $\Phi\left(2^{10} .2^{4} . S_{5}, 2\right) \backslash\left\{2^{10} .2^{4}\right\}$ and if $P_{2}=2^{10} .2^{4}$, then $|C|=2$ and $C={ }_{G} C(17)$.

Let $2^{6} .2^{5} \in \Phi\left(M_{4}, 2\right)$, so that $N_{M_{4}}\left(2^{6} .2^{5}\right)=N\left(2^{6} .2^{5}\right)=2^{6} .2^{5} . S_{6}$ and $N_{E}\left(2^{6} .2^{5}\right)=$ $N_{M_{4} .2}\left(2^{6} .2^{5}\right)=2^{6} .2^{5} \cdot\left(S_{6} \times 2\right)$. We may take

$$
\Phi\left(2^{6} .2^{5} . S_{6}, 2\right)=\left\{2^{6} .2^{5}, 2^{6} \cdot 2^{5} \cdot 2^{3},\left(2^{6} .2^{5} \cdot 2^{3}\right)^{*}, 2^{6} \cdot 2^{5} \cdot 2^{3} \cdot 2\right\}
$$

and in addition, $N_{2^{6} .2^{5} . S_{6}}(R)=R . S_{3}$ or $R$ according as $R \in\left\{2^{6} .2^{5} .2^{3},\left(2^{6} .2^{5} .2^{3}\right)^{*}\right\}$ or $R=2^{6} .2^{5} .2^{3} .2$ and $N_{N_{E}\left(2^{6} .2^{5}\right)}(R)=N_{2^{6} .2^{5} . S_{6}}(R) .2$ for each $R \in \Phi\left(2^{6} .2^{5} . S_{6}, 2\right)$.

Let $C^{\prime}: 1<2 \times 2_{+}^{1+8}<2^{6} .2^{5}<2^{6} .2^{5} .2^{3} .2$ and $g\left(C^{\prime}\right): 1<2 \times 2_{+}^{1+8}<2^{6} .2^{5}<$ $\left(2^{6} .2^{5} \cdot 2^{3}\right)^{*}<2^{6} \cdot 2^{5} \cdot 2^{3} .2$. Then $N\left(C^{\prime}\right)=N\left(g\left(C^{\prime}\right)\right), N_{E}\left(C^{\prime}\right)=N_{E}\left(g\left(C^{\prime}\right)\right)=N\left(C^{\prime}\right) .2$ and we may suppose $C \notin_{G}\left\{C^{\prime}, g\left(C^{\prime}\right)\right\}$. It follows that if $P_{1}=2 \times 2_{+}^{1+8}$, then

$$
C \in_{G}\{C(j): 15 \leq j \leq 20\} .
$$

Case (2.4) Let $2^{5+8} .2^{2} \in \Phi\left(M_{5}, 2\right)$, and $\sigma: 1<Q=2^{5+8}<2^{5+8} .2^{2}$. Since $N_{E}\left(2^{5+8} .2^{2}\right)=N_{M_{5} .2}\left(2^{5+8} \cdot 2^{2}\right)$, it follows that we may suppose $C \notin \mathcal{R}^{-}\left(\sigma, 2^{5+8}\right) \cup$ 
$\mathcal{R}^{0}\left(\sigma, 2^{5+8}\right)$, so we may suppose $P_{1} \neq_{G} 2^{5+8} .2$ and if $P_{1}=2^{5+8}$ with $|C| \geq 2$, then $P_{2} \neq_{G} 2^{5+8} \cdot 2^{2}$.

If $2^{5+8} .2 \in \Phi\left(M_{5}, 2\right)$, then $N\left(2^{5+8} .2\right)=N_{M_{5}}\left(2^{5+8} .2\right)=2^{5+8} \cdot 2 . A_{6}, N_{E}\left(2^{5+8} .2\right)=$ $N_{M_{5} .2}\left(2^{5+8} .2\right)=N\left(2^{5+8} .2\right) .2$ and we may take

$$
\Phi\left(2^{5+8} \cdot 2 \cdot A_{6}, 2\right)=\left\{2^{5+8} \cdot 2,2^{10} \cdot 2^{2} \cdot 2^{4}, 2^{5+8} \cdot 2^{3}, S\right\} .
$$

In addition, $N_{2^{5+8} .2 . A_{6}}(R)=N_{M_{5}}(R)$ and $N_{M_{5} .2}(R)=N_{M_{5}}(R) .2 \leq N_{M_{5} .2}\left(2^{5+8} .2\right)$ for each $R \in \Phi\left(2^{5+8} \cdot 2 \cdot A_{6}, 2\right)$.

Let $R \in\left\{2^{10} .2^{2} \cdot 2^{4}, 2^{5+8} .2^{3}\right\}$, and let $\sigma(R): 1<2^{5+8}<Q=2^{5+8} .2<R$. Then we may suppose $C \notin \mathcal{R}^{-}\left(\sigma(R), 2^{5+8} .2\right) \cup \mathcal{R}^{0}\left(\sigma(R), 2^{5+8} .2\right)$.

If $\left(2 \times 2_{+}^{1+8}\right) \cdot 2 \cdot 2^{4} \in \Phi\left(M_{5}, 2\right)$, then

$$
\begin{gathered}
N_{E}\left(\left(2 \times 2_{+}^{1+8}\right) \cdot 2 \cdot 2^{4}\right)=N_{M_{5} \cdot 2}\left(\left(2 \times 2_{+}^{1+8}\right) \cdot 2 \cdot 2^{4}\right)=N\left(\left(2 \times 2_{+}^{1+8}\right) \cdot 2 \cdot 2^{4}\right) \cdot 2 \\
N\left(\left(2 \times 2_{+}^{1+8}\right) \cdot 2 \cdot 2^{4}\right)=N_{M_{5}}\left(\left(2 \times 2_{+}^{1+8}\right) \cdot 2 \cdot 2^{4}\right)=\left(2 \times 2_{+}^{1+8}\right) \cdot 2 \cdot 2^{4} \cdot\left(S_{3} \times S_{3}\right) \text { and we may take } \\
\Phi\left(\left(2 \times 2_{+}^{1+8}\right) \cdot 2 \cdot 2^{4} \cdot\left(S_{3} \times S_{3}\right), 2\right)=\left\{\left(2 \times 2_{+}^{1+8}\right) \cdot 2 \cdot 2^{4}, 2^{10} \cdot 2^{2} \cdot 2^{4}, 2^{5+8} \cdot D_{8}, S\right\} .
\end{gathered}
$$

In addition, $N_{\left(2 \times 2_{+}^{1+8}\right) \cdot 2 \cdot 2^{4} .\left(S_{3} \times S_{3}\right)}(R)=N_{M_{5}}(R)$ and

$$
N_{M_{5} .2}(R)=N_{M_{5}}(R) .2 \leq N_{M_{5} .2}\left(\left(2 \times 2_{+}^{1+8}\right) .2 .2^{4}\right)
$$

for each $R \in \Phi\left(\left(2 \times 2_{+}^{1+8}\right) \cdot 2.2^{4} .\left(S_{3} \times S_{3}\right), 2\right)$.

Let $R \in\left\{2^{5+8} . D_{8}, S\right\}$, and $\sigma(R): 1<2^{5+8}<Q=\left(2 \times 2_{+}^{1+8}\right) \cdot 2.2^{4}<R$. Then we may suppose $C \notin \mathcal{R}^{-}\left(\sigma(R),\left(2 \times 2_{+}^{1+8}\right) .2 .2^{4}\right) \cup \mathcal{R}^{0}\left(\sigma(R),\left(2 \times 2_{+}^{1+8}\right) .2 .2^{4}\right)$.

Let $C^{\prime}: 1<2^{5+8}<2^{5+8} .2<S$ and $g\left(C^{\prime}\right): 1<2^{5+8}<\left(2 \times 2_{+}^{1+8}\right) .2 .2^{4}<2^{10} .2^{2} .2^{4}<$ $S$. Then (5.3) holds, and we may suppose $C \notin_{G}\left\{C^{\prime}, g\left(C^{\prime}\right)\right\}$. It follows that if $P_{1}=2^{5+8}$, then $C \in_{G}\{C(i): 21 \leq i \leq 24\}$.

Remark 5.2 Let $\hat{G}$ be a covering group of $G=\mathrm{Fi}_{22}$, $\rho$ a faithful linear character of $Z(\hat{G})$ and $\hat{B}$ a block of $\hat{G}$ covering the block $B(\rho)$ containing $\rho$. If $D(\hat{B}) \neq O_{p}(Z(\hat{G}))$ and $p=2,3$, then

$$
\sum_{C \in \mathcal{R}(G) / G}(-1)^{|C|} \mathrm{k}\left(N_{\hat{G}}(C), \hat{B}, d, \rho,[r]\right)=\sum_{C \in \mathcal{R}^{0}(G) / G}(-1)^{|C|} \mathrm{k}\left(N_{\hat{G}}(C), \hat{B}, d, \rho,[r]\right)
$$

for all integers $d, u \geq 0$.

The proof of the Remark is the same as that of Lemma 5.1, since $N\left(C^{\prime}\right)=N\left(g\left(C^{\prime}\right)\right)$ implies $N_{\hat{G}}\left(C^{\prime}\right)=N_{\hat{G}}\left(g\left(C^{\prime}\right)\right)$.

\section{Uno's invariant conjecture for $\mathrm{Fi}_{22}$}

Let $N(C)$ be the normalizer of a radical $p$-chain $C$. If $N(C)$ is a maximal subgroup of $\mathrm{Fi}_{22}$, then the character table of $N(C)$ can be found in the library of character tables 
distributed with GAP. If this is not the case, we construct a "useful" description of $N(C)$ and attempt to compute directly its character table using MAGMA.

If $N(C)$ is soluble, we construct a power-conjugate presentation for $N(C)$ and use this presentation to obtain the character table.

If $N(C)$ is insoluble, we construct faithful permutation representations for $N(C)$ and use these as input to the character table construction function. We employ two strategies to obtain faithful representations of $N(C)$.

1. Construct the actions of $N(C)$ on the cosets of soluble subgroups of $N(C)$.

2. Construct the orbits of $N(C)$ on the underlying set of $\mathrm{Fi}_{22}$; for the stabilizer of an orbit representative, construct the action of $N(C)$ on its cosets.

In several cases, however, none of the representations constructed was of sufficiently small degree to allow us to construct the required character table.

In these cases, we directly calculate the character table of $N(C)$ as follows: first calculate the character tables of some subgroups and quotient groups of $N(C)$; next induce or lift these characters to $N(C)$, so the liftings and the irreducible characters from the induction form a partial character table $T$ of $N(C)$; finally decompose the remaining inductions or the tensor products of the inductions using the table $T$.

The tables listing degrees of irreducible characters referenced in the proof of Theorem 6.1 are available electronically [5].

Theorem 6.1 Let $B$ be a p-block of $G=\mathrm{Fi}_{22}$ with positive defect. Then $B$ satisfies the Uno's invariant conjecture.

Proof: Let $E=\operatorname{Aut}(G)=\mathrm{Fi}_{22} .2$ and we may suppose $D(B)$ is non-cyclic, so that $B=B_{0}$ by Lemma 4.2 .

Case (1) Suppose $p=5$ and let $C=C(2), C^{\prime}=C(3)$, so that $N(C) \simeq 5: 4 \times S_{5}$, $N_{E}(C)=N(C) \times 2, N\left(C^{\prime}\right) \simeq 5: 4 \times 5: 4$ and $N_{E}\left(C^{\prime}\right)=N\left(C^{\prime}\right) \times 2$. It follows by $(2.6)$ that for all integers $u, d$

$$
\mathrm{k}\left(N(C), B_{0}, d, u,[r]\right)=\mathrm{k}\left(N\left(C^{\prime}\right), B_{0}, d, u,[r]\right) .
$$

Now $N(D(B))=N(C(4)) \simeq 5^{2}: 4 S_{4}, N_{E}(D(B))=5^{2}: 4 S_{4} \times 2$, and so

$$
\mathrm{k}\left(G, B_{0}, d, u,[r]\right)=\mathrm{k}\left(N(B(D)), B_{0}, d, u,[r]\right)= \begin{cases}10 & \text { if } d=2, u=2 \text { and } r=1, \\ 10 & \text { if } d=2, u=2 \text { and } r=2, \\ 0 & \text { otherwise. }\end{cases}
$$

This proves the theorem when $p=5$.

Case (2) Suppose $p=3$, so that Dade's invariant conjecture is equivalent to Uno's invariant conjecture. We set $\mathrm{k}(i, d, u)=\mathrm{k}\left(N(C(i)), B_{0}, d, u\right)$ for integers $i, d, u$.

First, we consider the radical 3-chains $C(j)$ with $\mathrm{d}(N(C(j)))=7$, so that $2 \leq j \leq 5$. The values $\mathrm{k}(i, d, u)$ are given in Table 5 . 


\begin{tabular}{|r||r|r|r|r|r|r|r|r|}
\hline Defect d & 7 & 7 & 6 & 6 & 5 & 5 & 4 & otherwise \\
\hline Value u & 2 & 1 & 2 & 1 & 2 & 1 & 2 & otherwise \\
\hline \hline $\mathrm{k}(2, \mathrm{~d}, \mathrm{u})$ & 36 & 18 & 18 & 0 & 18 & 0 & 6 & 0 \\
\hline $\mathrm{k}(3, \mathrm{~d}, \mathrm{u})$ & 36 & 18 & 15 & 12 & 18 & 0 & 0 & 0 \\
\hline $\mathrm{k}(4, \mathrm{~d}, \mathrm{u})$ & 36 & 18 & 15 & 12 & 18 & 18 & 0 & 0 \\
\hline $\mathrm{k}(5, \mathrm{~d}, \mathrm{u})$ & 36 & 18 & 18 & 0 & 18 & 18 & 6 & 0 \\
\hline
\end{tabular}

Table 5: Values of $\mathrm{k}(i, d, u)$ when $p=3$ and $\mathrm{d}(N(C(i)))=7$

It follows that

$$
\sum_{i=2}^{5}(-1)^{|C(i)|} \mathrm{k}\left(N(C(i)), B_{0}, d, u\right)=0 .
$$

Next we consider the chain $C(j)$ with $\mathrm{d}(N(C(j)))=9$, so that $j=1$ or $6 \leq j \leq 16$. The values $\mathrm{k}(i, d, u)$ are given in Table 6 .

\begin{tabular}{|r||r|r|r|r|r|r|r|r|r|r|r|r|}
\hline Defect d & 9 & 9 & 8 & 8 & 7 & 7 & 6 & 6 & 5 & 5 & 4 & otherwise \\
\hline Value u & 2 & 1 & 2 & 1 & 2 & 1 & 2 & 1 & 2 & 1 & 2 & otherwise \\
\hline $\mathrm{k}(1, \mathrm{~d}, \mathrm{u})$ & 12 & 6 & 19 & 2 & 6 & 2 & 1 & 2 & 6 & 0 & 2 & 0 \\
\hline $\mathrm{k}(6, \mathrm{~d}, \mathrm{u})$ & 12 & 6 & 19 & 2 & 16 & 12 & 1 & 2 & 6 & 0 & 0 & 0 \\
\hline $\mathrm{k}(7, \mathrm{~d}, \mathrm{u})=\mathrm{k}(9, \mathrm{~d}, \mathrm{u})$ & 0 & 18 & 0 & 12 & 0 & 46 & 0 & 3 & 0 & 0 & 0 & 0 \\
\hline $\mathrm{k}(8, \mathrm{~d}, \mathrm{u})=\mathrm{k}(10, \mathrm{~d}, \mathrm{u})$ & 0 & 18 & 0 & 12 & 0 & 13 & 0 & 3 & 0 & 0 & 0 & 0 \\
\hline $\mathrm{k}(11, \mathrm{~d}, \mathrm{u})=\mathrm{k}(15, \mathrm{~d}, \mathrm{u})$ & 0 & 18 & 0 & 21 & 0 & 13 & 0 & 18 & 0 & 3 & 0 & 0 \\
\hline $\mathrm{k}(12, \mathrm{~d}, \mathrm{u})$ & 12 & 6 & 22 & 8 & 6 & 2 & 6 & 12 & 6 & 6 & 2 & 0 \\
\hline $\mathrm{k}(13, \mathrm{~d}, \mathrm{u})$ & 12 & 6 & 22 & 8 & 16 & 12 & 6 & 12 & 6 & 0 & 0 & 0 \\
\hline $\mathrm{k}(16, \mathrm{~d}, \mathrm{u})=\mathrm{k}(14, \mathrm{~d}, \mathrm{u})$ & 0 & 18 & 0 & 21 & 0 & 46 & 0 & 18 & 0 & 0 & 0 & 0 \\
\hline
\end{tabular}

Table 6: Values of $\mathrm{k}(i, d, u)$ when $p=3$ and $\mathrm{d}(N(C(i)))=9$

It follows that

$$
\sum_{i=1,6 \leq i \leq 16}(-1)^{|C(i)|} \mathrm{k}\left(N(C(i)), B_{0}, d, u\right)=0
$$

and the theorem follows.

Case (3) First, we consider the 2-chains $C(j)$ such that the defect $\mathrm{d}(N(C(j)))=15$, so that $j \in\{10,11,12,13,15,18,19,20\}$. In this case, each element of $N_{E}(C) \backslash N(C)$ stabilizes each character of $\operatorname{Irr}(N(C))$. The values $\mathrm{k}(i, d, u)$ are given in Table 7 .

It follows that

$$
\sum_{i \in\{11,13,15,19\}} \mathrm{k}\left(N(C(i)), B_{0}, d, u\right)=\sum_{i \in\{10,12,18,20\}} \mathrm{k}\left(N(C(i)), B_{0}, d, u\right) .
$$

Next we consider the radical 2-chains $C(j)$ such that the defect $\mathrm{d}(N(C(j)))=17$, so that $j \in\{1,14,16,17,21,22,23,24\}$. The non-zero values $\mathrm{k}(i, d, u)$ are given in Table 8. 


\begin{tabular}{|r||r|r|r|r|r|r|r|r|r|}
\hline Defect d & 15 & 14 & 13 & 12 & 11 & 10 & 9 & 6 & otherwise \\
\hline Value $\mathrm{u}$ & 2 & 2 & 2 & 2 & 2 & 2 & 2 & 2 & otherwise \\
\hline \hline $\mathrm{k}(10, \mathrm{~d}, \mathrm{u})$ & 32 & 16 & 4 & 12 & 2 & 0 & 0 & 1 & 0 \\
\hline $\mathrm{k}(11, \mathrm{~d}, \mathrm{u})$ & 32 & 16 & 12 & 20 & 4 & 0 & 0 & 0 & 0 \\
\hline $\mathrm{k}(12, \mathrm{~d}, \mathrm{u})$ & 32 & 32 & 60 & 28 & 20 & 4 & 0 & 0 & 0 \\
\hline $\mathrm{k}(13, \mathrm{~d}, \mathrm{u})$ & 32 & 32 & 52 & 20 & 18 & 4 & 0 & 0 & 0 \\
\hline $\mathrm{k}(15, \mathrm{~d}, \mathrm{u})$ & 32 & 56 & 12 & 20 & 6 & 8 & 2 & 1 & 0 \\
\hline $\mathrm{k}(18, \mathrm{~d}, \mathrm{u})$ & 32 & 56 & 44 & 28 & 12 & 8 & 2 & 0 & 0 \\
\hline $\mathrm{k}(19, \mathrm{~d}, \mathrm{u})$ & 32 & 72 & 92 & 52 & 28 & 12 & 2 & 0 & 0 \\
\hline $\mathrm{k}(20, \mathrm{~d}, \mathrm{u})$ & 32 & 72 & 60 & 44 & 22 & 12 & 2 & 0 & 0 \\
\hline
\end{tabular}

Table 7: Values of $\mathrm{k}(i, d, u)$ when $p=2$ and $\mathrm{d}(N(C(i)))=15$

\begin{tabular}{|r||r|r|r|r|r|r|r|r|r|r|r|r|r|r|r|r|}
\hline Defect d & 17 & 16 & 15 & 14 & 14 & 13 & 13 & 12 & 12 & 11 & 11 & 10 & 10 & 9 & 9 & 6 \\
\hline Value u & 2 & 2 & 2 & 2 & 1 & 2 & 1 & 2 & 1 & 2 & 1 & 2 & 1 & 2 & 1 & 2 \\
\hline \hline $\mathrm{k}(1, \mathrm{~d}, \mathrm{u})$ & 16 & 12 & 2 & 4 & 4 & 3 & 6 & 9 & 0 & 2 & 0 & 1 & 0 & 0 & 2 & 1 \\
\hline $\mathrm{k}(14, \mathrm{~d}, \mathrm{u})$ & 16 & 12 & 2 & 4 & 4 & 2 & 2 & 0 & 0 & 0 & 0 & 1 & 0 & 0 & 0 & 0 \\
\hline $\mathrm{k}(16, \mathrm{~d}, \mathrm{u})$ & 16 & 12 & 10 & 20 & 4 & 10 & 6 & 14 & 4 & 8 & 4 & 3 & 0 & 1 & 2 & 1 \\
\hline $\mathrm{k}(17 \mathrm{~d}, \mathrm{u})$ & 16 & 12 & 10 & 24 & 4 & 11 & 2 & 9 & 4 & 2 & 0 & 3 & 0 & 0 & 0 & 0 \\
\hline $\mathrm{k}(21, \mathrm{~d}, \mathrm{u})$ & 16 & 12 & 18 & 10 & 4 & 18 & 2 & 4 & 0 & 0 & 0 & 0 & 4 & 0 & 0 & 0 \\
\hline $\mathrm{k}(22, \mathrm{~d}, \mathrm{u})$ & 16 & 12 & 18 & 10 & 4 & 19 & 6 & 13 & 0 & 2 & 0 & 0 & 4 & 0 & 2 & 0 \\
\hline $\mathrm{k}(23, \mathrm{~d}, \mathrm{u})$ & 16 & 12 & 26 & 26 & 4 & 26 & 6 & 26 & 4 & 8 & 4 & 2 & 4 & 1 & 2 & 0 \\
\hline $\mathrm{k}(24, \mathrm{~d}, \mathrm{u})$ & 16 & 12 & 26 & 30 & 4 & 27 & 2 & 21 & 4 & 2 & 0 & 2 & 4 & 0 & 0 & 0 \\
\hline
\end{tabular}

Table 8: Values of $\mathrm{k}(i, d, u)$ when $p=2$ and $\mathrm{d}(N(C(i)))=17$

It follows that

$$
\sum_{i \in\{1,17,21,23\}} \mathrm{k}\left(N(C(i)), B_{0}, d, u\right)=\sum_{i \in\{14,16,22,24\}} \mathrm{k}\left(N(C(i)), B_{0}, d, u\right) .
$$

Now we consider the radical 2-chains $C(j)$ such that the defect $\mathrm{d}(N(C(j)))=16$, so that $2 \leq j \leq 9$. The non-zero values $\mathrm{k}(i, d, u)$ are given in Table 9 .

It follows that

$$
\sum_{i \in\{3,5,7,9\}} \mathrm{k}\left(N(C(i)), B_{0}, d, u\right)=\sum_{i \in\{2,4,6,8\}} \mathrm{k}\left(N(C(i)), B_{0}, d, u\right) .
$$

Thus the theorem follows by (6.3), (6.4) and (6.5). This completes the proof. 


\begin{tabular}{|r||r|r|r|r|r|r|r|r|r|r|r|r|r|r|r|r|r|}
\hline Defect d & 16 & 16 & 15 & 15 & 14 & 14 & 13 & 13 & 12 & 12 & 11 & 11 & 10 & 10 & 9 & 9 & 6 \\
\hline Value u & 2 & 1 & 2 & 1 & 2 & 1 & 2 & 1 & 2 & 1 & 2 & 1 & 2 & 1 & 2 & 1 & 2 \\
\hline \hline $\mathrm{k}(2, \mathrm{~d}, \mathrm{u})$ & 8 & 8 & 4 & 4 & 4 & 0 & 12 & 4 & 5 & 4 & 6 & 4 & 6 & 2 & 2 & 0 & 2 \\
\hline $\mathrm{k}(3, \mathrm{~d}, \mathrm{u})$ & 8 & 8 & 4 & 4 & 4 & 0 & 12 & 12 & 13 & 12 & 8 & 12 & 6 & 2 & 2 & 4 & 2 \\
\hline $\mathrm{k}(4, \mathrm{~d}, \mathrm{u})$ & 8 & 8 & 4 & 4 & 4 & 0 & 14 & 6 & 13 & 10 & 6 & 0 & 4 & 2 & 0 & 0 & 0 \\
\hline $\mathrm{k}(5, \mathrm{~d}, \mathrm{u})$ & 8 & 8 & 4 & 4 & 4 & 0 & 10 & 2 & 5 & 2 & 0 & 0 & 4 & 2 & 0 & 0 & 0 \\
\hline $\mathrm{k}(6, \mathrm{~d}, \mathrm{u})$ & 8 & 8 & 4 & 4 & 12 & 12 & 10 & 2 & 13 & 10 & 4 & 0 & 4 & 0 & 0 & 0 & 0 \\
\hline $\mathrm{k}(7, \mathrm{~d}, \mathrm{u})$ & 8 & 8 & 4 & 4 & 12 & 12 & 12 & 4 & 13 & 12 & 10 & 4 & 6 & 0 & 2 & 0 & 0 \\
\hline $\mathrm{k}(8, \mathrm{~d}, \mathrm{u})$ & 8 & 8 & 4 & 4 & 12 & 12 & 12 & 12 & 21 & 20 & 12 & 12 & 6 & 8 & 2 & 4 & 0 \\
\hline $\mathrm{k}(9, \mathrm{~d}, \mathrm{u})$ & 8 & 8 & 4 & 4 & 12 & 12 & 14 & 6 & 21 & 18 & 10 & 0 & 4 & 8 & 0 & 0 & 0 \\
\hline
\end{tabular}

Table 9: Values of $\mathrm{k}(i, d, u)$ when $p=2$ and $\mathrm{d}(N(C(i)))=16$

\section{Uno's projective invariant conjecture for $2 . \mathrm{Fi}_{22}$}

Let $C$ be a radical $p$-chain of $\mathrm{Fi}_{22}$. The character tables of $N_{2 . \mathrm{Fi}_{22}}(C)$ and $N_{2 . \mathrm{Fi}_{22.2}}(C)$ can either be found in the library of character tables distributed with GAP or computed directly using Magma as in Section 6.

Let $H=N_{2 . \mathrm{Fi}_{22}}(C)$ or $N_{2 . \mathrm{Fi}_{22.2}}(C)$ and let $\xi$ be the faithful linear character of $Z\left(2 . \mathrm{Fi}_{22}\right)$. Denote by $\operatorname{Irr}(H \mid \xi)$ the subset of $\operatorname{Irr}(H)$ consisting of characters covering $\xi$. The tables listing degrees of irreducible characters referenced in the proof of Theorem 7.1 are available electronically [5].

Theorem 7.1 Let $B$ be a p-block of $G=2 . \mathrm{Fi}_{22}$ with $D(B) \neq O_{p}(G)$. Then $B$ satisfies Uno's projective invariant conjecture.

Proof: We may suppose $B$ has a non-cyclic defect group. Let $N(C)=N_{G}(C)$ for each $C \in \mathcal{R}\left(\mathrm{Fi}_{22}\right)$ and let $E=G .2=2 . \mathrm{Fi}_{22} .2$.

Case (1) Suppose $p=5$, so that $B=B_{1}$ given by Lemma 4.2. Thus

$$
\mathrm{k}\left(G, B_{1}, d, u, \xi\right)=\mathrm{k}\left(N(C(4)), B_{1}, d, u, \xi\right)= \begin{cases}2 & \text { if } d=2, u=2 \text { and } r=2, \\ 2 & \text { if } d=2, u=2 \text { and } r=1, \\ 8 & \text { if } d=2, u=1 \text { and } r=2, \\ 8 & \text { if } d=2, u=1 \text { and } r=1, \\ 0 \quad \text { otherwise, }\end{cases}
$$

and when $j=1$ or 2 ,

$$
\mathrm{k}\left(N(C(j)), B_{1}, d, u, \xi,[r]\right)= \begin{cases}1 & \text { if } d=2, u=2 \text { and } r=1 \\ 24 & \text { if } d=2, u=1 \text { and } r=1 \\ 0 & \text { otherwise }\end{cases}
$$

This implies the theorem when $p=5$. 
Case (2) Suppose $p=3$, so that the projective invariant conjecture of Uno is equivalent to that of Dade. By Lemma $4.2, B=B_{1}$ and we set $\mathrm{k}(j, d, u)=$ $\mathrm{k}(N(C(j)), B, d, u, \xi)$.

We first consider the radical 3-chains $C(j)$ with $\mathrm{d}(N(C(j)))=7$, so that $2 \leq j \leq 5$. The values $\mathrm{k}(j, d, u)$ are given in Table 10 .

\begin{tabular}{|r||r|r|r|r|r|r|r|r|r|}
\hline Defect d & 7 & 7 & 6 & 6 & 5 & 5 & 4 & 4 & otherwise \\
\hline Value u & 2 & 1 & 2 & 1 & 2 & 1 & 2 & 1 & otherwise \\
\hline \hline $\mathrm{k}(2, \mathrm{~d}, \mathrm{u})$ & 4 & 50 & 3 & 6 & 0 & 18 & 2 & 4 & 0 \\
\hline $\mathrm{k}(3, \mathrm{~d}, \mathrm{u})$ & 4 & 50 & 1 & 26 & 0 & 18 & 0 & 0 & 0 \\
\hline $\mathrm{k}(4, \mathrm{~d}, \mathrm{u})$ & 4 & 50 & 1 & 26 & 6 & 30 & 0 & 0 & 0 \\
\hline $\mathrm{k}(5, \mathrm{~d}, \mathrm{u})$ & 4 & 50 & 3 & 6 & 6 & 30 & 2 & 4 & 0 \\
\hline
\end{tabular}

Table 10: Values of $\mathrm{k}\left(N(C(i)), B_{1}, d, u, \xi\right)$ when $p=3$ and $\mathrm{d}(N(C(i)))=7$

It follows that

$$
\sum_{i=2}^{5}(-1)^{|C(i)|} \mathrm{k}\left(N(C(i)), B_{1}, d, u, \xi\right)=0 .
$$

Next we consider the chain $C(j)$ with $\mathrm{d}(N(C(j)))=9$, so that $j=1$ or $6 \leq j \leq 16$. The values $\mathrm{k}(i, d, u)$ are given in Table 11 .

\begin{tabular}{|r||r|r|r|r|r|r|r|r|r|r|}
\hline Defect d & 9 & 9 & 8 & 8 & 7 & 7 & 6 & 6 & 5 & otherwise \\
\hline Value u & 2 & 1 & 2 & 1 & 2 & 1 & 2 & 1 & 1 & otherwise \\
\hline \hline $\mathrm{k}(1, \mathrm{~d}, \mathrm{u})$ & 4 & 14 & 3 & 18 & 1 & 0 & 1 & 2 & 6 & 0 \\
\hline $\mathrm{k}(6, \mathrm{~d}, \mathrm{u})$ & 4 & 14 & 3 & 18 & 4 & 24 & 1 & 2 & 6 & 0 \\
\hline $\mathrm{k}(7, \mathrm{~d}, \mathrm{u})=\mathrm{k}(9, \mathrm{~d}, \mathrm{u})$ & 0 & 18 & 0 & 12 & 0 & 46 & 0 & 3 & 0 & 0 \\
\hline $\mathrm{k}(8, \mathrm{~d}, \mathrm{u})=\mathrm{k}(10, \mathrm{~d}, \mathrm{u})$ & 0 & 18 & 0 & 12 & 0 & 13 & 0 & 3 & 0 & 0 \\
\hline $\mathrm{k}(11, \mathrm{~d}, \mathrm{u})=\mathrm{k}(15, \mathrm{~d}, \mathrm{u})$ & 0 & 18 & 0 & 21 & 0 & 13 & 0 & 18 & 3 & 0 \\
\hline $\mathrm{k}(12, \mathrm{~d}, \mathrm{u})$ & 4 & 14 & 4 & 26 & 1 & 0 & 0 & 18 & 12 & 0 \\
\hline $\mathrm{k}(13, \mathrm{~d}, \mathrm{u})$ & 4 & 14 & 4 & 26 & 4 & 24 & 0 & 18 & 6 & 0 \\
\hline $\mathrm{k}(16, \mathrm{~d}, \mathrm{u})=\mathrm{k}(14, \mathrm{~d}, \mathrm{u})$ & 0 & 18 & 0 & 21 & 0 & 46 & 0 & 18 & 0 & 0 \\
\hline
\end{tabular}

Table 11: Values of $\mathrm{k}\left(N(C(i)), B_{1}, d, u, \xi\right)$ when $p=3$ and $\mathrm{d}(N(C(i)))=9$

It follows that

$$
\sum_{i=1,6 \leq i \leq 16}(-1)^{|C(i)|} \mathrm{k}\left(N(C(i)), B_{1}, d, u, \xi\right)=0
$$

and the theorem follows.

Case (3) Suppose $p=2$, so that by Lemma $4.2, B=B_{0}$ or $B_{1}$. If $B=B_{1}$, then

$$
\mathrm{k}\left(G, B_{1}, d, u, \xi\right)=\mathrm{k}\left(N(C(2)), B_{1}, d, u, \xi\right)= \begin{cases}2 & \text { if } d=2 \text { and } u=1 \\ 0 & \text { otherwise }\end{cases}
$$


and the theorem follows when $B=B_{1}$.

Set $\mathrm{k}(j, d, u)=\mathrm{k}\left(N(C(j)), B_{0}, d, u, \xi\right)$. We first consider the radical 2-chains $C(j)$ such that the defect $\mathrm{d}(N(C(j)))=16$, so that $j \in\{10,11,12,13,15,18,19,20\}$. The values $\mathrm{k}(i, d, u)$ are given in Table 12 .

\begin{tabular}{|r||r|r|r|r|r|r|r|r|r|r|}
\hline Defect d & 14 & 13 & 13 & 12 & 12 & 11 & 11 & 10 & 8 & otherwise \\
\hline Value u & 1 & 2 & 1 & 2 & 1 & 2 & 1 & 2 & 1 & otherwise \\
\hline \hline $\mathrm{k}(10, \mathrm{~d}, \mathrm{u})$ & 16 & 4 & 8 & 2 & 8 & 0 & 4 & 1 & 4 & 0 \\
\hline $\mathrm{k}(11, \mathrm{~d}, \mathrm{u})$ & 16 & 12 & 8 & 2 & 8 & 0 & 4 & 1 & 0 & 0 \\
\hline $\mathrm{k}(12, \mathrm{~d}, \mathrm{u})$ & 16 & 12 & 40 & 2 & 24 & 4 & 4 & 1 & 0 & 0 \\
\hline $\mathrm{k}(13, \mathrm{~d}, \mathrm{u})$ & 16 & 4 & 40 & 2 & 24 & 4 & 4 & 1 & 0 & 0 \\
\hline $\mathrm{k}(15, \mathrm{~d}, \mathrm{u})$ & 16 & 4 & 8 & 2 & 40 & 0 & 8 & 0 & 4 & 0 \\
\hline $\mathrm{k}(18, \mathrm{~d}, \mathrm{u})$ & 16 & 12 & 8 & 10 & 40 & 0 & 8 & 0 & 0 & 0 \\
\hline $\mathrm{k}(19, \mathrm{~d}, \mathrm{u})$ & 16 & 12 & 40 & 10 & 56 & 4 & 8 & 0 & 0 & 0 \\
\hline $\mathrm{k}(20, \mathrm{~d}, \mathrm{u})$ & 16 & 4 & 40 & 2 & 56 & 4 & 8 & 0 & 0 & 0 \\
\hline
\end{tabular}

Table 12: Values of $\mathrm{k}\left(N(C(j)), B_{0}, d, u, \xi\right)$ when $p=2$ and $\mathrm{d}(N(C(i)))=16$

It follows that

$$
\sum_{i \in\{11,13,15,19\}} \mathrm{k}\left(N(C(i)), B_{0}, d, u, \xi\right)=\sum_{i \in\{10,12,18,20\}} \mathrm{k}\left(N(C(i)), B_{0}, d, u, \xi\right) .
$$

Next we consider the radical 2-chains $C(j)$ such that the defect $\mathrm{d}(N(C(j)))=18$, so that $j \in\{1,14,16,17,21,22,23,24\}$. The values $\mathrm{k}(i, d, u)$ are given in Table 13 .

\begin{tabular}{|r||r|r|r|r|r|r|r|r|r|r|}
\hline Defect d & 14 & 13 & 13 & 12 & 12 & 11 & 11 & 10 & 8 & otherwise \\
\hline Value u & 2 & 2 & 1 & 2 & 1 & 2 & 1 & 1 & 1 & otherwise \\
\hline \hline $\mathrm{k}(1, \mathrm{~d}, \mathrm{u})$ & 8 & 2 & 12 & 4 & 12 & 2 & 0 & 2 & 4 & 0 \\
\hline $\mathrm{k}(14, \mathrm{~d}, \mathrm{u})$ & 8 & 2 & 12 & 2 & 0 & 2 & 0 & 0 & 0 & 0 \\
\hline $\mathrm{k}(16, \mathrm{~d}, \mathrm{u})$ & 24 & 2 & 16 & 0 & 20 & 2 & 4 & 2 & 4 & 0 \\
\hline $\mathrm{k}(17 \mathrm{~d}, \mathrm{u})$ & 24 & 2 & 16 & 2 & 8 & 2 & 0 & 0 & 0 & 0 \\
\hline $\mathrm{k}(21, \mathrm{~d}, \mathrm{u})$ & 8 & 2 & 28 & 2 & 0 & 0 & 4 & 0 & 0 & 0 \\
\hline $\mathrm{k}(22, \mathrm{~d}, \mathrm{u})$ & 8 & 2 & 28 & 4 & 12 & 0 & 4 & 2 & 0 & 0 \\
\hline $\mathrm{k}(23, \mathrm{~d}, \mathrm{u})$ & 24 & 2 & 32 & 0 & 20 & 0 & 8 & 2 & 0 & 0 \\
\hline $\mathrm{k}(24, \mathrm{~d}, \mathrm{u})$ & 24 & 2 & 32 & 2 & 8 & 0 & 4 & 0 & 0 & 0 \\
\hline
\end{tabular}

Table 13: Values of $\mathrm{k}\left(N(C(i)), B_{0}, d, u, \xi\right)$ when $p=2$ and $\mathrm{d}(N(C(i)))=18$ It follows that

$$
\sum_{i \in\{1,17,21,23\}} \mathrm{k}\left(N(C(i)), B_{0}, d, u, \xi\right)=\sum_{i \in\{14,16,22,24\}} \mathrm{k}\left(N(C(i)), B_{0}, d, u, \xi\right) .
$$


Now we consider the radical 2-chains $C(j)$ such that the defect $\mathrm{d}(N(C(j)))=17$, so that $2 \leq j \leq 9$. The values $\mathrm{k}(i, d, u)$ are given in Table 14 .

\begin{tabular}{|r||r|r|r|r|r|r|r|}
\hline Defect $\mathrm{d}$ & 14 & 13 & 12 & 11 & 10 & 7 & otherwise \\
\hline Value $\mathrm{u}$ & 1 & 1 & 1 & 1 & 1 & 1 & otherwise \\
\hline \hline $\mathrm{k}(2, \mathrm{~d}, \mathrm{u})$ & 16 & 12 & 20 & 8 & 2 & 2 & 0 \\
\hline $\mathrm{k}(3, \mathrm{~d}, \mathrm{u})$ & 16 & 28 & 28 & 8 & 6 & 2 & 0 \\
\hline $\mathrm{k}(4, \mathrm{~d}, \mathrm{u})$ & 16 & 28 & 8 & 8 & 0 & 0 & 0 \\
\hline $\mathrm{k}(5, \mathrm{~d}, \mathrm{u})$ & 16 & 12 & 0 & 8 & 0 & 0 & 0 \\
\hline $\mathrm{k}(6, \mathrm{~d}, \mathrm{u})$ & 16 & 44 & 0 & 4 & 0 & 0 & 0 \\
\hline $\mathrm{k}(7, \mathrm{~d}, \mathrm{u})$ & 16 & 44 & 20 & 4 & 2 & 0 & 0 \\
\hline $\mathrm{k}(8, \mathrm{~d}, \mathrm{u})$ & 16 & 60 & 28 & 12 & 6 & 0 & 0 \\
\hline $\mathrm{k}(9, \mathrm{~d}, \mathrm{u})$ & 16 & 60 & 8 & 12 & 0 & 0 & 0 \\
\hline
\end{tabular}

Table 14: Values of $\mathrm{k}\left(N(C(i)), B_{0}, d, u, \xi\right)$ when $p=2$ and $\mathrm{d}(N(C(i)))=17$

It follows that

$$
\sum_{i \in\{3,5,7,9\}} \mathrm{k}\left(N(C(i)), B_{0}, d, u, \xi\right)=\sum_{i \in\{2,4,6,8\}} \mathrm{k}\left(N(C(i)), B_{0}, d, u, \xi\right) .
$$

The theorem follows.

\section{Uno's projective conjecture for $3 . \mathrm{Fi}_{22}$}

Let $C$ be a radical $p$-chain of $\mathrm{Fi}_{22}$ and $N_{3 . \mathrm{Fi}_{22}}(C)=3 . N_{\mathrm{Fi}_{22}}(C)$. The character table of $N_{3 . \mathrm{Fi}_{22}}(C)$ can either be found in the library of character tables distributed with GAP or computed directly using MAgma as in Section 6.

Let $H=N_{3 . \mathrm{Fi}_{22}}(C)$ and let $\zeta_{1}$ and let $\zeta_{2}$ be the faithful linear characters of $Z\left(3 . \mathrm{Fi}_{22}\right)$. Denote by $\operatorname{Irr}\left(H \mid \zeta_{i}\right)$ the subset of $\operatorname{Irr}(H)$ consisting of characters covering $\zeta_{i}$. The tables listing degrees of irreducible characters referenced in the proof of Theorem 8.1 are available electronically [5].

Theorem 8.1 Let $B$ be a p-block of $G=3 . \mathrm{Fi}_{22}$ with $D(B) \neq O_{p}(G)$. Then $B$ satisfies Uno's projective conjecture.

Proof: We may suppose $B$ has a non-cyclic defect group and let $N(C)=N_{G}(C)$ for each $C \in \mathcal{R}\left(\mathrm{Fi}_{22}\right)$.

Case (1) Suppose $p=5$, so that $B=B_{1}$ or $B_{2}$. By [9, pp. 156-159], $\mathrm{k}\left(G, B_{\ell}, d, \zeta_{\ell}\right)=$ $\sum_{u \geq 0} \mathrm{k}\left(G, B_{0}\left(\mathrm{Fi}_{22}\right), d, u\right)$ for each $\ell$. 
If $R$ is a non-trivial radical $p$-subgroup of $G$, then $R=5$ or $R=5^{2} \in \operatorname{Syl}_{5}(G)$, and $N_{G}(R)=3 \times N_{\mathrm{Fi}_{22}}(R)$, so that for $i \geq 2, N(C(i))=3 \times N_{\mathrm{Fi}_{22}}(C(i))$, where $C(i)$ is a radical chain of $\mathrm{Fi}_{22}$ given by Table 3 . Thus

$$
\mathrm{k}\left(N(C(i)), B_{\ell}, d, \zeta_{\ell}\right)=\sum_{u \geq 0} \mathrm{k}\left(N_{\mathrm{Fi}_{22}}(C), B_{0}\left(\mathrm{Fi}_{22}\right), d, u\right)
$$

and the proof (1) of Theorem 6.1 can be applied here for blocks $B=B_{1}$ and $B_{2}$. This proves the theorem when $p=5$.

Case (2) Suppose $p=3$, so that $B=B_{0}, B_{1}$ or $B_{2}$ given by Lemma 4.2 (c).

Suppose $B=B_{1}$ or $B_{2}$. Then $D(B) \simeq 3^{2}, N(D(B))=N(C(2))$ and the theorem follows by Remark 2.1 and

$$
\mathrm{k}\left(G, B, d, \zeta_{\ell}\right)=\mathrm{k}\left(N_{G}(C(2)), B, d, \zeta_{\ell}\right)= \begin{cases}6 & \text { if } d=2, \\ 0 & \text { otherwise }\end{cases}
$$

for $\ell=1,2$.

Suppose $B=B_{0}$ and set $\mathrm{k}(j, d)=\mathrm{k}\left(N(C(j)), B_{0}, d, \zeta_{\ell}\right)$. We first consider the radical 3-chains $C(j)$ with $\mathrm{d}(N(C(j)))=8$, so that $2 \leq j \leq 5$. The values $\mathrm{k}(j, d)$ are given in Table 15.

\begin{tabular}{|r||r|r|r|r|}
\hline Defect d & 7 & 6 & 5 & otherwise \\
\hline \hline $\mathrm{k}(2, \mathrm{~d})$ & 54 & 15 & 12 & 0 \\
\hline $\mathrm{k}(3, \mathrm{~d})$ & 54 & 15 & 0 & 0 \\
\hline $\mathrm{k}(4, \mathrm{~d})$ & 54 & 33 & 0 & 0 \\
\hline $\mathrm{k}(5, \mathrm{~d})$ & 54 & 33 & 12 & 0 \\
\hline
\end{tabular}

Table 15: Values of $\mathrm{k}\left(N(C(i)), B_{0}, d, \zeta_{\ell}\right)$ when $p=3$ and $\mathrm{d}(N(C(i)))=8$

It follows that

$$
\sum_{i=2}^{5}(-1)^{|C(i)|} \mathrm{k}\left(N(C(i)), B_{0}, d, \zeta_{\ell}\right)=0 .
$$

Next we consider the chain $C(j)$ with $\mathrm{d}(N(C(j)))=10$, so that $j=1$ or $6 \leq j \leq 16$. The values $\mathrm{k}(i, d)$ are given in Table 16 .

It follows that

$$
\sum_{i=1,6 \leq i \leq 16}(-1)^{|C(i)|} \mathrm{k}\left(N(C(i)), B_{0}, d, \zeta_{\ell}\right)=0
$$

and the theorem follows.

Case (3) Suppose $p=2$, so that $B=B_{1}$ or $B_{2}$.

Let $M \in\left\{2^{5+8} .\left(3 \times A_{5}\right) \cdot 2,2^{6} \cdot S_{6}(2),\left(2 \times 2_{+}^{1+8}\right): U_{4}(2): 2\right\}$ be a subgroup of $\mathrm{Fi}_{22}$ and $H=3 . M$ the preimage of $M$ in $G$. Let $S$ be a Sylow 2-subgroup of $H, H_{1}=O_{3}(Z(H))$ and $H_{2}=\langle[H, H], S\rangle$, where $[H, H]$ is the commutator subgroup of $H$. Then $H=$ $H_{1} \times H_{2}$ and $H_{2} \simeq M$, so that $H \simeq 3 \times M$. Let

$$
\Omega=\{3,4,6,7,8,9,10,11,12,13,15,16,17,18,19,20,23,24\}
$$




\begin{tabular}{|r||r|r|r|r|}
\hline Defect d & 7 & 6 & 4 & otherwise \\
\hline \hline $\mathrm{k}(1, \mathrm{~d})$ & 27 & 14 & 2 & 0 \\
\hline $\mathrm{k}(6, \mathrm{~d})=\mathrm{k}(13, \mathrm{~d})$ & 27 & 5 & 0 & 0 \\
\hline $\mathrm{k}(7, \mathrm{~d})=\mathrm{k}(9, \mathrm{~d})=\mathrm{k}(14, \mathrm{~d})=\mathrm{k}(16, \mathrm{~d})$ & 9 & 2 & 0 & 0 \\
\hline $\mathrm{k}(8, \mathrm{~d})=\mathrm{k}(10, \mathrm{~d})$ & 9 & 2 & 1 & 0 \\
\hline $\mathrm{k}(11, \mathrm{~d})=\mathrm{k}(15, \mathrm{~d})$ & 9 & 2 & 0 & 0 \\
\hline $\mathrm{k}(12, \mathrm{~d}, \mathrm{u})$ & 27 & 14 & 0 & 0 \\
\hline
\end{tabular}

Table 16: Values of $\mathrm{k}\left(N(C(i)), B_{0}, d, \zeta_{\ell}\right)$ when $p=3$ and $\mathrm{d}(N(C(i)))=10$

and $i \in \Omega$. Then $N_{G}(C(i))$ is a subgroup of some $H=3 . M$ with $N_{\mathrm{Fi}_{22}}(C(i)) \leq M$, so that we may suppose

$$
N_{G}(C(i))=3 \times N_{\mathrm{Fi}_{22}}(C(i)) .
$$

It follows that for any $i \in \Omega, j, \ell \in\{1,2\}$,

$$
\mathrm{k}\left(N_{G}(C(i)), B_{j}, d, \zeta_{\ell}\right)=\sum_{u \geq 0} \mathrm{k}\left(N_{\mathrm{Fi}_{22}}(C(i)), B_{0}\left(\mathrm{Fi}_{22}\right), d, u\right)
$$

and $\mathrm{k}\left(N_{G}(C(i)), B_{j}, d, \zeta_{\ell}\right)$ can be obtained by knowing $\mathrm{k}\left(N_{\mathrm{Fi}_{22}}(C(i)), B_{0}\left(\mathrm{Fi}_{22}\right), d, u\right)$, which is given in the proof of Theorem 6.1. Since $\mathrm{k}\left(N(C(i)), B_{j}, d, \zeta_{\ell}\right)$ is independent of the choices of $j$ and $\ell$, we set $\mathrm{k}(i, d)=\mathrm{k}\left(N(C(i)), B, d, \zeta_{\ell}\right)$ for integers $i, d$.

Now we consider the 2-chains $C(j)$ such that the defect $\mathrm{d}(N(C(j))=15$, so that $j \in\{10,11,12,13,15,18,19,20\} \subseteq \Omega$. Thus if $C=C(j)$, then $N(C) \simeq 3 \times N_{\mathrm{Fi}_{22}}(C)$ and $\mathrm{k}(j, d)$ is given by $(8.1)$. It follows by $(6.3)$ that

$$
\sum_{j \in\{11,13,15,19\}} \mathrm{k}\left(N(C(j)), B, d, \zeta_{\ell}\right)=\sum_{j \in\{10,12,18,20\}} \mathrm{k}\left(N(C(j)), B, d, \zeta_{\ell}\right)
$$

for each $\ell$.

Next we consider the radical 2-chains $C(j)$ such that the defect $\mathrm{d}(N(C(j)))=17$, so that $j \in\{1,14,16,17,21,22,23,24\}$. The values $\mathrm{k}(i, d)$ are given in Table 17 .

It follows that

$$
\sum_{i \in\{1,17,21,23\}} \mathrm{k}\left(N(C(i)), B, d, \zeta_{\ell}\right)=\sum_{i \in\{14,16,22,24\}} \mathrm{k}\left(N(C(i)), B, d, \zeta_{\ell}\right) .
$$

Now we consider the radical 2-chains $C(j)$ such that the defect $\mathrm{d}(N(C(j)))=16$, so that $2 \leq j \leq 9$. The values $\mathrm{k}(i, d)$ are given in Table 18 .

It follows that

$$
\sum_{i \in\{3,5,7,9\}} \mathrm{k}\left(N(C(i)), B, d, \zeta_{\ell}\right)=\sum_{i \in\{2,4,6,8\}} \mathrm{k}\left(N(C(i)), B, d, \zeta_{\ell}\right) .
$$

The theorem follows. 


\begin{tabular}{|r||r|r|r|r|r|r|r|r|r|r|r|}
\hline Defect d & 17 & 16 & 15 & 14 & 13 & 12 & 11 & 10 & 9 & 6 & otherwise \\
\hline \hline $\mathrm{k}(1, \mathrm{~d})$ & 16 & 12 & 2 & 4 & 3 & 9 & 2 & 1 & 0 & 1 & 0 \\
\hline $\mathrm{k}(14, \mathrm{~d})$ & 16 & 12 & 2 & 4 & 4 & 0 & 0 & 1 & 0 & 0 & 0 \\
\hline $\mathrm{k}(16, \mathrm{~d})$ & 16 & 12 & 10 & 24 & 16 & 18 & 12 & 3 & 3 & 1 & 0 \\
\hline $\mathrm{k}(17 \mathrm{~d})$ & 16 & 12 & 10 & 28 & 13 & 13 & 2 & 3 & 0 & 0 & 0 \\
\hline $\mathrm{k}(21, \mathrm{~d})$ & 16 & 12 & 18 & 10 & 20 & 4 & 0 & 0 & 0 & 0 & 0 \\
\hline $\mathrm{k}(22, \mathrm{~d})$ & 16 & 12 & 18 & 10 & 19 & 13 & 2 & 0 & 0 & 0 & 0 \\
\hline $\mathrm{k}(23, \mathrm{~d})$ & 16 & 12 & 26 & 30 & 32 & 30 & 12 & 6 & 3 & 0 & 0 \\
\hline $\mathrm{k}(24, \mathrm{~d})$ & 16 & 12 & 26 & 34 & 29 & 25 & 2 & 6 & 0 & 0 & 0 \\
\hline
\end{tabular}

Table 17: Values of $\mathrm{k}\left(N(C(i)), B, d, \zeta_{\ell}\right)$ when $p=2$ and $\mathrm{d}(N(C(i)))=17$

\begin{tabular}{|r||r|r|r|r|r|r|r|r|r|r|}
\hline Defect d & 16 & 15 & 14 & 13 & 12 & 11 & 10 & 9 & 6 & otherwise \\
\hline \hline $\mathrm{k}(2, \mathrm{~d})$ & 16 & 8 & 4 & 16 & 9 & 10 & 4 & 2 & 2 & 0 \\
\hline $\mathrm{k}(3, \mathrm{~d})$ & 16 & 8 & 4 & 24 & 25 & 20 & 8 & 6 & 2 & 0 \\
\hline $\mathrm{k}(4, \mathrm{~d})$ & 16 & 8 & 4 & 20 & 23 & 6 & 6 & 0 & 0 & 0 \\
\hline $\mathrm{k}(5, \mathrm{~d})$ & 16 & 8 & 4 & 12 & 7 & 0 & 2 & 0 & 0 & 0 \\
\hline $\mathrm{k}(6, \mathrm{~d})$ & 16 & 8 & 24 & 12 & 23 & 4 & 4 & 0 & 0 & 0 \\
\hline $\mathrm{k}(7, \mathrm{~d})$ & 16 & 8 & 24 & 16 & 25 & 14 & 6 & 2 & 0 & 0 \\
\hline $\mathrm{k}(8, \mathrm{~d})$ & 16 & 8 & 24 & 24 & 41 & 24 & 14 & 6 & 0 & 0 \\
\hline $\mathrm{k}(9, \mathrm{~d})$ & 16 & 8 & 24 & 20 & 39 & 10 & 12 & 0 & 0 & 0 \\
\hline
\end{tabular}

Table 18: Values of $\mathrm{k}\left(N(C(i)), B, d, \zeta_{\ell}\right)$ when $p=2$ and $\mathrm{d}(N(C(i)))=16$

\section{Uno's projective conjecture for $6 . \mathrm{Fi}_{22}$}

Let $C$ be a radical $p$-chain of $\mathrm{Fi}_{22}$ and $N_{6 . \mathrm{Fi}_{22}}(C)=6 . N_{\mathrm{Fi}_{22}}(C)$. The character table of $N_{6 . \mathrm{Fi}_{22}}(C)$ can either be found in the library of character tables distributed with GAP or computed directly using MAGMA or GAP as in Section 6.

Let $H=N_{6 . \mathrm{Fi}_{22}}(C)$ and let $\eta_{1}$ and $\eta_{2}$ be the faithful linear characters of $Z\left(6 . \mathrm{Fi}_{22}\right)$. Denote by $\operatorname{Irr}\left(H \mid \eta_{i}\right)$ the subset of $\operatorname{Irr}(H)$ consisting of characters covering $\eta_{i}$. The tables listing degrees of irreducible characters referenced in the proof of Theorem 9.1 are available electronically [5].

Theorem 9.1 Let $B$ be a p-block of $G=6 . \mathrm{Fi}_{22}$ with $D(B) \neq O_{p}(G)$. Then $B$ satisfies Uno's projective conjecture.

Proof: We may suppose $B$ has a non-cyclic defect group. Let $N(C)=N_{G}(C)$ for each $C \in \mathcal{R}\left(\mathrm{Fi}_{22}\right)$.

Case (1) Suppose $p=5$, so that $B=B_{1}$ or $B_{2}$. Then

$$
\mathrm{k}\left(N(C(4)), B, d, \eta_{\ell},[r]\right)=\sum_{u \geq 0} \mathrm{k}\left(N_{\mathrm{Fi}_{22}}(C), B_{1}\left(2 . \mathrm{Fi}_{22}\right), d, u, \xi,[r]\right)
$$


and the theorem follows by

$$
\mathrm{k}\left(G, B, d, \eta_{\ell},[r]\right)=\mathrm{k}\left(N(C(4)), B, d, \eta_{\ell},[r]\right)= \begin{cases}10 & \text { if } d=2 \text { and } r=1, \\ 10 & \text { if } d=2 \text { and } r=2, \\ 0 & \text { otherwise. }\end{cases}
$$

Case (2) Suppose $p=3$, so that $B=B_{1}$. Then for each $\ell, j \in\{1,2\}$, the degrees of characters in $\operatorname{Irr}\left(N(C(i)) \mid \eta_{\ell}\right)$ are the same as that of characters in $\operatorname{Irr}\left(N_{3 . \mathrm{Fi}_{22}}(C(i)) \mid\right.$ $\left.\zeta_{j}\right)$ and so

$$
\mathrm{k}\left(N(C(i)), B_{1}, d, \eta_{\ell}\right)=\mathrm{k}\left(N_{3 . \mathrm{Fi}_{22}}(C(i)), B_{0}\left(3 . \mathrm{Fi}_{22}\right), d, \zeta_{j}\right),
$$

except when $i \in\{1,2,5,12\}$, in which case the values $\mathrm{k}(i, d)=\mathrm{k}\left(N(C(i)), B_{1}, d, \eta_{\ell}\right)$ are given in Table 19.

\begin{tabular}{|r||r|r|r|r|r|}
\hline Defect d & 7 & 6 & 5 & 4 & otherwise \\
\hline \hline $\mathrm{k}(1, \mathrm{~d})$ & 27 & 5 & 0 & 2 & 0 \\
\hline $\mathrm{k}(2, \mathrm{~d})$ & 54 & 15 & 3 & 0 & 0 \\
\hline $\mathrm{k}(5, \mathrm{~d})$ & 54 & 33 & 3 & 0 & 0 \\
\hline $\mathrm{k}(12, \mathrm{~d})$ & 54 & 5 & 0 & 0 & 0 \\
\hline
\end{tabular}

Table 19: Values of $\mathrm{k}\left(N(C(i)), B_{1}, d, \zeta_{\ell}\right)$ when $p=3$

It follows by Tables 15, 16 and 19 that

$$
\sum_{i=1}^{14}(-1)^{|C(i)|} \mathrm{k}\left(N(C(i)), B_{0}, d, \zeta_{\ell}\right)=0
$$

Case (3) Suppose $p=2$, so that $B=B_{1}$ or $B_{2}$. The proof is similar to that of Theorem 8.1.

Let $M \in\left\{2^{5+8} \cdot\left(3 \times A_{5}\right) \cdot 2,2^{6} . S_{6}(2),\left(2 \times 2_{+}^{1+8}\right): U_{4}(2): 2\right\}$ be a subgroup of $\mathrm{Fi}_{22}$ and $H=6 . M$ the preimage of $M$ in $G$. If $H_{1}=O_{3}(Z(H))$ and $H_{2}$ is the subgroup of $H$ generated by both the commutator subgroup $[H, H]$ and a Sylow 2-subgroup of $H$, then $H=H_{1} \times H_{2}$ and $H_{2} \simeq 2 . M \leq 2$. $\mathrm{Fi}_{22}$, so that $H \simeq 3 \times 2 . M$. Let

$$
\Omega=\{3,4,6,7,8,9,10,11,12,13,15,16,17,18,19,20,23,24\}
$$

and $i \in \Omega$. Then $N_{G}(C(i))$ is a subgroup of $H=6 . M$ for some $M$, and $N_{\mathrm{Fi}_{22}}(C(i)) \leq$ $M$, so that $N_{G}(C(i))=3 \times N_{2 . \mathrm{Fi}_{22}}(C(i))$ and for any $i \in \Omega, j, \ell \in\{1,2\}$,

$$
\mathrm{k}\left(N_{G}(C(i)), B_{j}, d, \eta_{\ell}\right)=\sum_{u \geq 0} \mathrm{k}\left(N_{2 . \mathrm{Fi}_{22}}(C(i)), B_{0}\left(2 . \mathrm{Fi}_{22}\right), d, u, \xi\right)
$$

where $\xi$ is the restriction of $\eta_{\ell}$ to $O_{2}(Z(G))$. Thus $\mathrm{k}\left(N_{G}(C(i)), B_{j}, d, \eta_{\ell}\right)$ can be obtained by knowing $\mathrm{k}\left(N_{2 . \mathrm{Fi}_{22}}(C(i)), B_{0}\left(2 . \mathrm{Fi}_{22}\right), d, u, \xi\right)$, which is given in the proof of Theorem 7.1 .

Since $\mathrm{k}\left(N(C(i)), B_{j}, d, \eta_{\ell}\right)$ is independent of the choices of $j$ and $\ell$, we set $\mathrm{k}(i, d)=$ $\mathrm{k}\left(N(C(i)), B, d, \eta_{\ell}\right)$ for integers $i, d$. 
Consider the 2-chains $C(j)$ such that the defect $\mathrm{d}(N(C(j))=16$, so that $j \in$ $\{10,11,12,13,15,18,19,20\} \subseteq \Omega$. Thus if $C=C(j)$, then $N(C) \simeq 3 \times N_{2 . \mathrm{Fi}_{22}}(C)$ and $\mathrm{k}(j, d)$ is given by $(9.1)$. It follows by $(7.1)$ that

$$
\sum_{j \in\{11,13,15,19\}} \mathrm{k}\left(N(C(j)), B, d, \eta_{\ell}\right)=\sum_{j \in\{10,12,18,20\}} \mathrm{k}\left(N(C(j)), B, d, \eta_{\ell}\right)
$$

for each $\ell$.

Next we consider the radical 2-chains $C(j)$ such that the defect $\mathrm{d}(N(C(j)))=18$, so that $j \in\{1,14,16,17,21,22,23,24\}$. The values $\mathrm{k}(i, d)$ are given in Table 20 .

\begin{tabular}{|r||r|r|r|r|r|r|r|}
\hline Defect d & 14 & 13 & 12 & 11 & 10 & 8 & otherwise \\
\hline \hline $\mathrm{k}(1, \mathrm{~d})$ & 8 & 6 & 8 & 6 & 2 & 4 & 0 \\
\hline $\mathrm{k}(14, \mathrm{~d})$ & 8 & 6 & 2 & 6 & 0 & 0 & 0 \\
\hline $\mathrm{k}(16, \mathrm{~d})$ & 24 & 18 & 20 & 6 & 2 & 4 & 0 \\
\hline $\mathrm{k}(17 \mathrm{~d})$ & 24 & 18 & 10 & 2 & 0 & 0 & 0 \\
\hline $\mathrm{k}(21, \mathrm{~d})$ & 8 & 22 & 2 & 4 & 0 & 0 & 0 \\
\hline $\mathrm{k}(22, \mathrm{~d})$ & 8 & 22 & 8 & 4 & 2 & 0 & 0 \\
\hline $\mathrm{k}(23, \mathrm{~d})$ & 24 & 34 & 20 & 8 & 2 & 0 & 0 \\
\hline $\mathrm{k}(24, \mathrm{~d})$ & 24 & 34 & 10 & 4 & 0 & 0 & 0 \\
\hline
\end{tabular}

Table 20: Values of $\mathrm{k}\left(N(C(i)), B, d, \eta_{\ell}\right)$ when $p=2$ and $\mathrm{d}(N(C(i)))=18$

It follows that

$$
\sum_{i \in\{1,17,21,23\}} \mathrm{k}\left(N(C(i)), B, d, \eta_{\ell}\right)=\sum_{i \in\{14,16,22,24\}} \mathrm{k}\left(N(C(i)), B, d, \eta_{\ell}\right) .
$$

Now we consider the radical 2-chains $C(j)$ such that the defect $\mathrm{d}(N(C(j)))=17$, so that $2 \leq j \leq 9$. The values $\mathrm{k}(i, d)$ are given in Table 21 .

\begin{tabular}{|r||r|r|r|r|r|r|r|}
\hline Defect d & 14 & 13 & 12 & 11 & 10 & 7 & otherwise \\
\hline \hline $\mathrm{k}(2, \mathrm{~d})$ & 16 & 12 & 20 & 4 & 2 & 2 & 0 \\
\hline $\mathrm{k}(3, \mathrm{~d})$ & 16 & 28 & 28 & 8 & 6 & 2 & 0 \\
\hline $\mathrm{k}(4, \mathrm{~d})$ & 16 & 28 & 8 & 8 & 0 & 0 & 0 \\
\hline $\mathrm{k}(5, \mathrm{~d})$ & 16 & 12 & 4 & 0 & 0 & 0 & 0 \\
\hline $\mathrm{k}(6, \mathrm{~d})$ & 16 & 44 & 4 & 0 & 0 & 0 & 0 \\
\hline $\mathrm{k}(7, \mathrm{~d})$ & 16 & 44 & 20 & 4 & 2 & 0 & 0 \\
\hline $\mathrm{k}(8, \mathrm{~d})$ & 16 & 60 & 28 & 12 & 6 & 0 & 0 \\
\hline $\mathrm{k}(9, \mathrm{~d})$ & 16 & 60 & 8 & 12 & 0 & 0 & 0 \\
\hline
\end{tabular}

Table 21: Values of $\mathrm{k}\left(N(C(i)), B, d, \eta_{\ell}\right)$ when $p=2$ and $\mathrm{d}(N(C(i)))=17$ 
It follows that

$$
\sum_{i \in\{3,5,7,9\}} \mathrm{k}\left(N(C(i)), B, d, \eta_{\ell}\right)=\sum_{i \in\{2,4,6,8\}} \mathrm{k}\left(N(C(i)), B, d, \eta_{\ell}\right) .
$$

The theorem follows.

\section{Acknowledgment}

The authors thank Thomas Breuer for constructing the character tables of several maximal subgroups of the covering groups of $\mathrm{Fi}_{22}$.

\section{References}

[1] J.L. Alperin, Weights for finite groups, in The Arcata Conference on Representations of Finite Groups, Proceedings of Symposia in Pure Mathematics 47 (1987), 369-379.

[2] J. An and C. W. Eaton, Modular representation theory of blocks with trivial intersection defect groups, Algebra and Representation Theory, to appear.

[3] J. An and E.A. O'Brien, A local strategy to decide the Alperin and Dade conjectures, Journal of Algebra 189 (1997), 34-57.

[4] J. An and E.A. O'Brien, The Alperin and Dade conjectures for the Fischer simple group $\mathrm{Fi}_{23}$, International Journal of Algebra and Computation, 9 (1999), 621-670.

[5] J. An and E.A. O'Brien, Chain normalizers of the Fischer group $\mathrm{Fi}_{22}$, www.math.auckland.ac.nz/obrien/research/fi22/appendix.dvi

[6] ATLAS of Finite Group Representations, at http://for.mat.bham.ac.uk/atlas.

[7] W. Bosma, J. Cannon and C. Playoust, The Magma Algebra System I: The User Language, Journal of Symbolic Computation 24, (1997), 235-265.

[8] N. Burgoyne and C. Williamson, On a theorem of Borel and Tits for finite Chevalley groups, Archiv der Mathematik 27 (1976), 489-491.

[9] J.H. Conway, R.T. Curtis, S.P. Norton, R.A. Parker and R.A. Wilson, Atlas of finite groups, Clarendon Press, Oxford, 1985.

[10] F. Celler, C. R. Leedham-Green, S. H. Murray, A. C. Niemeyer and E.A. O'Brien, Generating random elements of a finite group, Communications in Algebra 23 (1995), 4931-4948.

[11] E.C. Dade, Counting Characters in Blocks, I, Inventions Mathematicae 109 (1992), 187-210.

[12] E.C. Dade, Counting characters in blocks, II.9, Representation theory of finite groups (Columbus, OH, 1995), Ohio State University Mathematical Research Institute Publications 6 de Gruyter, Berlin, 1997, pp. 45-59. 
[13] E.C. Dade, Counting Characters in Blocks with Cyclic Defect Groups, I, Journal of Algebra 186 (1996), 934-969.

[14] D. Flaass, 2-local subgroups of Fischer groups, Matematicheskie Zametki 35 (1984), 333-342.

[15] G. Hiss and K. Lux, Brauer Trees of Sporadic Groups. Oxford Science Publications, 1989.

[16] I.M. Isaacs and G. Navarro, New refinements of the McKay conjecture for arbitrary finite groups, Annals of Mathematics 156 (2002), 333-344.

[17] H. Nagao and Y. Tsushima, Representations of Finite Groups, Academic Press, Inc., Boston, MA, 1989.

[18] The GAP Team, GAP - Groups, Algorithms, and Programming, Version 4. Lehrstuhl D für Mathematik, RWTH Aachen and School of Mathematical and Computational Sciences, University of St Andrews, 2000.

[19] K. Uno, Conjectures on character degrees for the simple Thompson group, Osaka Journal of Mathematics, 41 (2004), 11-36.

[20] R.A. Wilson, Standard generators for sporadic simple groups, Journal of Algebra 184 (1996), 505-515.

[21] R.A. Wilson, On maximal subgroups of the Fischer group $\mathrm{Fi}_{22}$, Mathematical Proceedings of the Cambridge Philosophical Society 95 (1984), 197-222.

Department of Mathematics, University of Auckland, Auckland, New Zealand Email: an@math.auckland.ac.nz, obrien@math.auckland.ac.nz 